\title{
Graphene based functional devices: A short review
}

\author{
Rong Wang and Lijun Jiang* \\ Department of Electrical and Electronic Engineering, \\ The University of Hong Kong, Pokfulam Road, Hong Kong. \\ Xingang $\operatorname{Ren}^{\dagger}$ \\ Key Laboratory of Intelligent Computing \& Signal Processing, \\ Ministry of Education, Anhui University, Hefei 230039, China and \\ Department of Electrical and Electronic Engineering, \\ The University of Hong Kong, Pokfulam Road, Hong Kong. \\ Ze Yan \\ School of Instrumentation Science 85 Opto-electronics Engineering, Beihang University, Beijing 100191, China. \\ Wei E. I. Sha \\ Key Laboratory of Micro-nano Electronic Devices and Smart Systems of Zhejiang Province, \\ College of Information Science 86 Electronic Engineering, Zhejiang University, Hangzhou 310027, China. \\ Guangcun Shan \\ School of Instrumentation Science \& Opto-electronics Engineering, Beihang University, Beijing 100191, China. and \\ California NanoSystem Institute and Department of Chemistry $\&$ Biochemistry, \\ University of California, Los Angeles, CA 90095,USA
}

(Dated: October 19, 2018)

\begin{abstract}
Graphene is an ideal 2D material system bridging electronic and photonic devices. It also breaks the fundamental speed and size limits by electronics and photonics, respectively. Graphene offers multiple functions of signal transmission, emission, modulation, and detection in a broad band, high speed, compact size, and low loss. Here, we will have a brief view of graphene based functional devices at microwave, terahertz, and optical frequencies. Their fundamental physics and computational models will be discussed as well.
\end{abstract}

\section{INTRODUCTION}

Electron wavelength is much shorter than photon wavelength. Hence electronic devices exhibit compact sizes. Unfortunately, the electron transport is limited by its mobility or scattering events, which lowers its speed compared to photon transmission or propagation. On the other hand, the sizes of photonic devices are held back by the diffraction or half-wavelength limit. Surface plasmon polariton, which is an entangled state of electron and photon, is a promising candidate to achieve high speed and small size simultaneously. Unfortunately, high ohmic loss of metallic plasmon devices creates a fundamental hurdle for their potential applications.

Graphene, a conjugated carbon sheet arranged in a two-dimensional (2D) hexagonal lattice, allows multifunctions in signal emission, transmission, modulation, and detection, featured with the broad band, high speed, compact size, and particularly low loss. Compared to traditional materials such as silicon and III-V semiconductors, graphene demonstrates unique properties in its high electron mobility, large thermal conductivity, strong

\footnotetext{
* ljiang@eee.hku.hk

† xgren@ahu.edu.cn

$\ddagger$ gcshan@buaa.edu.cn
}

mechanical ductility, and high third-order optical nonlinearities. There are many reviews in literature about graphene photonics and plasmonics [1-7, 9-18, 25]. Here, we will present a brief review of graphene based functional devices at microwave, terahertz, and optical frequencies. The paper is organized as follows. The basic physical foundations and computational models of graphene will be discussed in Section 2 first. Then, the fabrication and relevant experimental results of graphene based functional devices at microwave frequencies will be introduced in Section 3. In Section 4, the application of graphene in dynamically altering the antenna characteristics is presented from terahertz to mid-infrared bands. Section 5 will present recent progresses on graphene modulators at terahertz and infrared bands. Then graphene detectors at terahertz frequencies will be discussed in details at Section 6. At the end, Section 7 will provide a brief summary for graphene based functional devices.

\section{PHYSICAL FOUNDATION AND THEORETICAL MODELING}

Graphene is a hexagonal lattice of carbon atoms arranged in a single atomic sheet. The electronic structure of graphene is essential to its electromagnetic responses. By using tight-binding model and creation and annihila- 


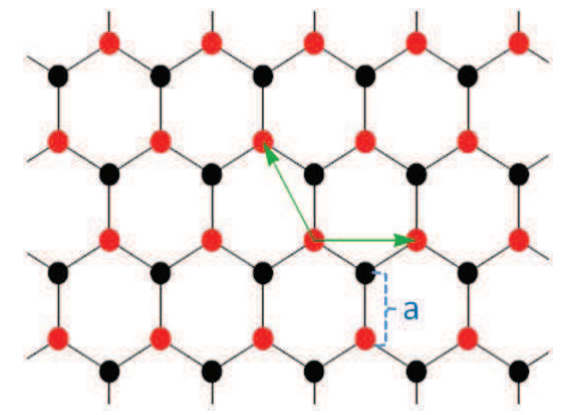

FIG. 1. Hexagonal lattice of graphene. Two atoms or sublattices per unit cell are denoted by red and black, respectively. The green arrows show two lattice vectors. The length of the nearest-neighbor bond is $a$.

tion operators, the Hamiltonian of graphene can be written as

$H=-t \sum_{i} a_{\mathbf{r}_{i}}^{+} b_{\mathbf{r}_{i}+\mathbf{e}_{1}}-t \sum_{i} a_{\mathbf{r}_{i}}^{+} b_{\mathbf{r}_{i}+\mathbf{e}_{2}}-t \sum_{i} a_{\mathbf{r}_{i}}^{+} b_{\mathbf{r}_{i}+\mathbf{e}_{3}}+$ h.c.

where $\mathbf{e}_{1}=(0, a), \mathbf{e}_{2}=\left(\frac{-\sqrt{3} a}{2}, \frac{-a}{2}\right), \mathbf{e}_{3}=\left(\frac{\sqrt{3} a}{2}, \frac{-a}{2}\right)$ are the three vectors pointing to three nearest-neighbor sites. $a$ is the length of the nearest-neighbor bonds. $t \approx 2.8$ $\mathrm{eV}$ is the nearest-neighbor hopping energy. The above Hamiltonian can be recasted into a series of summations in the momentum space, i.e.

$$
\begin{aligned}
& H=-t \sum_{\mathbf{k}} a_{\mathbf{k}}^{+} b_{\mathbf{k}}\left(e^{-i \mathbf{k} \cdot \mathbf{e}_{1}}+e^{-i \mathbf{k} \cdot \mathbf{e}_{2}}+e^{-i \mathbf{k} \cdot \mathbf{e}_{3}}\right)+h . c . \\
& =\sum_{\mathbf{k}}\left(\begin{array}{cc}
a_{\mathbf{k}}^{+} & b_{\mathbf{k}}^{+}
\end{array}\right)\left(\begin{array}{cc}
0 & H_{12}(\mathbf{k}) \\
H_{21}(\mathbf{k}) & 0
\end{array}\right)\left(\begin{array}{c}
a_{\mathbf{k}} \\
b_{\mathbf{k}}
\end{array}\right)
\end{aligned}
$$

where $H_{12}(\mathbf{k})=-t\left(e^{-i \mathbf{k} \cdot \mathbf{e}_{1}}+e^{-i \mathbf{k} \cdot \mathbf{e}_{2}}+e^{-i \mathbf{k} \cdot \mathbf{e}_{3}}\right)=H_{21}^{*}(\mathbf{k})$. The eigenvalues of Hamiltonian lead to the dispersion relation

$$
\begin{aligned}
& E(\mathbf{k})= \pm \sqrt{H_{12}(\mathbf{k}) H_{12}^{*}(\mathbf{k})} \\
& = \pm t \sqrt{1+4 \cos \left(\frac{\sqrt{3} a}{2} k_{x}\right) \cos \left(\frac{3 a}{2} k_{y}\right)+4 \cos ^{2}\left(\frac{\sqrt{3} a}{2} k_{x}\right)}
\end{aligned}
$$

The conduction and valance bands touch each other at the six Dirac points of the first Brillouin zone. Using Taylor expansion at the Dirac points, the dispersion relation of graphene is approximate to be linear, i.e.

$$
E(\mathbf{k})= \pm \hbar v_{F}|\mathbf{k}|, \quad \hbar v_{F}=3 a t / 2
$$

where $v_{F} \approx 10^{6} \mathrm{~m} / \mathrm{s}$ is the Fermi velocity.

The electron motion of graphene at the conduction band, which is also known as the intraband transition, can be described by the Boltzmann equation

$$
\frac{\partial f}{\partial t}+\frac{\partial \mathbf{p}}{\partial t} \cdot \frac{\partial E}{\partial \mathbf{p}} \frac{\partial f}{\partial E}=\frac{f_{0}-f}{\tau}
$$

where $f$ is a probability density function of electron defined in the $(\mathbf{r}, \mathbf{k})$-phase space. $E$ and $\mathbf{p}$ are the kinetic energy and momentum of electron. Here we use the relaxation time approximation and $\tau$ is the relaxation time. Moreover, we ignore the term $(\partial \mathbf{r} / \partial t)(\partial f / \partial \mathbf{r})$ in the Boltzmann equation, which is related to the diffusion current ignored for the conduction electrons. The above formulation can be rewritten as

$$
\frac{\partial f}{\partial t}+\mathbf{F} \cdot \mathbf{v} \frac{\partial f}{\partial E}=\frac{f_{0}-f}{\tau}
$$

where $\mathbf{v}$ is the group velocity and $\mathbf{F}=e \mathbf{E}$, where $\mathbf{E}$ is the electric field. Moreover, $E=\hbar \omega, \mathbf{p}=\hbar \mathbf{k}, \mathbf{v}=\partial \omega / \partial \mathbf{k}$. Using the Fourier transform, we arrive at

$$
\left(-i \omega+\frac{1}{\tau}\right) f=-\mathbf{F} \cdot \mathbf{v} \frac{\partial f}{\partial E}+\frac{f_{0}}{\tau}
$$

By multiplying Eq. (7) by $\mathbf{v}$ and then integrating it over $\mathbf{k}$ space, and we finally get

$$
\begin{aligned}
& \frac{g}{(2 \pi)^{2}}\left(-i \omega+\frac{1}{\tau}\right) \int e f \mathbf{v} d k_{x} d k_{y}= \\
& \frac{e g}{(2 \pi)^{2}} \int-\mathbf{F} \cdot(\mathbf{v v}) \frac{\partial f}{\partial E} d k_{x} d k_{y}
\end{aligned}
$$

where $g$ is degeneracy, which is equal to 4 for the graphene sheet considering spin degeneracy and two carbon atoms in a unit cell.

By using $\mathbf{J}=g /(2 \pi)^{2} \int e f \mathbf{v} d k_{x} d k_{y}$, we can get the expression for the conduction-band current

$$
\mathbf{J}=\frac{1}{-i \omega+\frac{1}{\tau}} \frac{e}{\pi^{2}} \int \mathbf{F} \cdot(\mathbf{v v}) \frac{-\partial f}{\partial E} d k_{x} d k_{y}
$$

Next, we consider the electrical impedance and ignore the magnetic field that is responsible for the Hall impedance, i.e.

$$
\sigma_{x x}^{\mathrm{intra}}=\frac{1}{-i \omega+\frac{1}{\tau}} \frac{e^{2}}{\pi^{2}} \int v_{x}^{2} \frac{-\partial f}{\partial E} d k_{x} d k_{y}=\sigma_{y y}^{\mathrm{int} r a}
$$

By using the approximate linear dispersion relation Eq. (4) and replacing $v_{x}^{2}$ with $v_{F}^{2} / 2$, in view of the fact $\partial f / \partial E$ is small except in the vicinity of Fermi-level. As a result, Eq. (10) can be rewritten as

$$
\begin{aligned}
\sigma_{x x}^{\text {intra }} & =\frac{1}{-i \omega+\frac{1}{\tau}} \frac{e^{2}}{\pi \hbar^{2}} \int \frac{-\partial f}{\partial E} E d E \\
& =\frac{-i}{\omega+i \frac{1}{\tau}} \frac{e^{2}}{\pi \hbar^{2}} \int_{0}^{\infty} E\left(\frac{\partial f(E)}{\partial E}-\frac{\partial f(-E)}{\partial E}\right) d E
\end{aligned}
$$

where $f=1 /\left(e^{\left(E-\mu_{c}\right) / k_{B} T}+1\right)$ is the Fermi-Dirac distribution, and $\mu_{c}$ is the chemical potential. The above agrees with the intraband transition part of Kubo formula [18]. The Eq. (11) is responsible for the imaginary part of the complex conductivity and thus plasmonic effects. 
The real part of the conductivity, which corresponds to the interband transition and generation of electron-hole pair, can be obtained by employing the kramers-kronig relation or Fermi's golden rule [19]. Finally, the complex surface conductivity is of the following form [18]

$$
\begin{aligned}
\sigma^{s}= & \frac{-i}{\omega+i \frac{1}{\tau}} \frac{e^{2}}{\pi \hbar^{2}} \int_{0}^{\infty} E\left(\frac{\partial f(E)}{\partial E}-\frac{\partial f(-E)}{\partial E}\right) d E+ \\
& i\left(\omega+i \frac{1}{\tau}\right) \frac{e^{2}}{\pi \hbar^{2}} \int_{0}^{\infty} \frac{f(-E)-f(E)}{(\omega+i / \tau)^{2}-4(E / \hbar)^{2}} d E
\end{aligned}
$$

When photon energy is larger than $2 \mu_{c}$, optical conductivity is dominated by the interband transition. As carrier concentration increases by the electrostatic doping of graphene such that the photon energy is less than $2 \mu_{c}$, intraband transition becomes dominant while interband transitions are suppressed due to the Pauli blocking.

There are two common scenarios to theoretically model graphene by classical computational electromagnetics algorithms. One approach is to use the effective bulk permittivity. The in-plane bulk permittivity of graphene is defined as $\varepsilon_{r}=1+i \sigma^{s} /\left(\omega \varepsilon_{0} d\right)$, where $d$ refers to the effective thickness of graphene sheet, and $\sigma^{s}$ is the surface conductivity calculated by the Kubo-formula of Eq. (12). The out-of-plane permittivity is chosen to be the dielectric permittivity of 2.5 [20]. Another approach is to adopt the surface conductivity directly; and implement corresponding surface impedance condition [21-23], i.e. $\mathbf{n} \times\left(\mathbf{H}_{1}-\mathbf{H}_{2}\right)=\sigma^{s} \mathbf{E}_{t}$, where $\mathbf{H}_{1}$ and $\mathbf{H}_{2}$ are the magnetic fields above and below the graphene sheet and $\mathbf{E}_{t}$ is the electric field tangential to the graphene sheet. More details regarding computational techniques can be found in [24].

\section{FABRICATION AND MICROWAVE DEVICES}

Tremendous efforts have been made over the last decade towards the fabrication of graphene, which, in general, can be categorized into three groups: (1) mechanical cleavage (MC), (2) epitaxial growth (EG), and (3) chemical vapor deposition (CVD). MC is realized by mechanically splitting bulk graphite into atomically thin single layer graphene with the aid of an adhesive tape. The first in-lab fabrication of single layer graphene was demonstrated by Novoselov et al. in 2005 [25] and the ballistic mobilities of up to $10^{6} \mathrm{~cm}^{2} \mathrm{~V}^{-1} \mathrm{~s}^{-1}$ were experimentally observed $[26,27]$. Although MC is favorable for high-quality graphene fabrication, the very limited size (often coexists with other multiple atomic layers) and the time-cost exfoliation process prevent the large-scale industrial production using this method. Therefore, the artificial synthesis of scaled-up single layer graphene has received great interests all over the world. EG, also known as growth on silicon carbide, utilizes high temperature (over $1000{ }^{\circ} \mathrm{C}$ ) to thermally evaporate silicon (0001) from silicon carbide and leaves graphene films on the surface $[28,29]$. The mobility ranging from $1 \times 10^{4} \mathrm{~cm}^{2} \mathrm{~V}^{-1} \mathrm{~s}^{-1}$ to $3 \times 10^{4} \mathrm{~cm}^{2} \mathrm{~V}^{-1} \mathrm{~s}^{-1}$ was reported on the EG-grown graphene surface measured at room temperature [30, 31]. Since silicon carbide has been widely used in high-speed electronics as a bottom substrate, the direct growth of single layer graphene on silicon carbide circumvents the additional substrate transfer process and benefits the graphene-integrated silicon carbide devices such as highfrequency transistors [32] and light emitting diodes [33]. However, due to the non self-limiting nature of thermal decomposition, it's difficult to generate pure single-layer carbon surface on silicon carbide surface and special care needs to be paid during the EG synthesis process. Furthermore, the high cost of silicon carbide wafers (more than 100 US dollar for a 4-inch wafer) limits the massive manufacture of graphene. In order to fulfil the low-cost and large-scale single layer graphene fabrication, CVD steps onto the stage, which, in principle, decomposes carbon atoms from hydrocarbons (methane, for example) and nucleates them on certain kinds of metal surfaces such as copper and finally forms an uniform and large area single layer graphene. The first large-area synthesis of single layer graphene using CVD was demonstrated in 2009 [34] and mobility at room temperature varying from $1.64 \times 10^{4} \mathrm{~cm}^{2} \mathrm{~V}^{-1} \mathrm{~s}^{-1}$ to $2.5 \times 10^{4} \mathrm{~cm}^{2} \mathrm{~V}^{-1} \mathrm{~s}^{-1}$ was obtained after the transfer of the single-layer graphene from the copper foil to the insulated substrate [35, 36]. Different from EG, CVD is almost self-contained. It means that the growth procedure will automatically pause when the entire catalytic metal surface is covered with single layer graphene. As a result, large area (over square decimeters) and uniform single layer graphene can be efficiently synthesized using this method. However, by virtue of the difference in thermal expansion coefficient between copper and graphene [34], defective wrinkles could occur on the graphene surface. They significantly degrade this two-dimensional material's quality, which can be seen from the much smaller mobility compared with the ones of MC-graphene. In order to provide costeffective as well as high-quality single layer graphene for massive production, further optimization and improvement need to be done for the CVD method.

Following the growth of graphene on copper foil in CVD chamber, the single layer graphene shall be transferred to the desired substrates with the aid of PMMA (Polymethyl Methacrylate) [37]. As illustrated in Fig. 2(a), this transfer process is composed of four steps: (1) A PMMA layer is firstly spin coated on top of graphene layer; (2) The supporting copper is etched away using $\mathrm{Cu}$ etchant; (3) The PMMA/graphene membrane is then attached to the target substrate such as the high-resistivity silicon; (4) The PMMA adhesive layer is dissolved in organic solvent and the graphene is eventually left on the target substrate. A distilled water rinse is suggested to get rid of the chemical residues during the transfer process. The adhesiveness between graphene and substrate could be further enhanced with several hour's baking so 
that the water molecules can fully evaporate.

A graphene-integrated circuit is ready for manufacture after the successful transfer process. Here, we take the graphene-embedded coplanar waveguide as an example and introduce the fabrication technologies utilized for electrodes deposition and graphene patterning [38]. As shown in Fig. 2(b), the deposition of coplanar waveguide electrodes (Process I) includes three stages: (1) Photo-lithography is applied to form the coplanar waveguide pattern on the photoresist layer; (2) Bi-metal layer $(20 \mathrm{~nm} \mathrm{Ti} / 100 \mathrm{~nm} \mathrm{Au})$ is then deposited on the graphene sheet using E-Beam evaporation; (3) The unwanted metal is removed during lift-off. Graphene patterning (Process II) is also achieved by three steps: (1) A pair of $48 \times 240 \mu \mathrm{m}^{2}$ Photoresist pattern cover on the graphene sheet between the signal line and the ground; (2) the graphene sheet that is not covered by photoresist is etched away by the oxygen plasma. Note that the plasma power cannot be too high to destroy the protection photoresist; (3) Photoresist is finally stripped with the acetone followed by distilled water rinse. Fig. 2(c) illustrates the layout of the graphene-embedded coplanar waveguide and the patterned graphene flakes can be clearly identified under scanning electron microscope (SEM) in Fig. 2(d).

The development of CVD enables the fabrication of large-area graphene with the size comparable to the wavelength of microwave frequencies and boosts the usage of graphene at microwave band. Before this, most of the works were focused on the simulation region, such as graphene antenna [39], orbital angular momentum modulator [40] and frequency selective surface [41]. The simulated graphene layer is normally treated as an impedance surface or an ultra-thin bulk layer based on Kubo for-
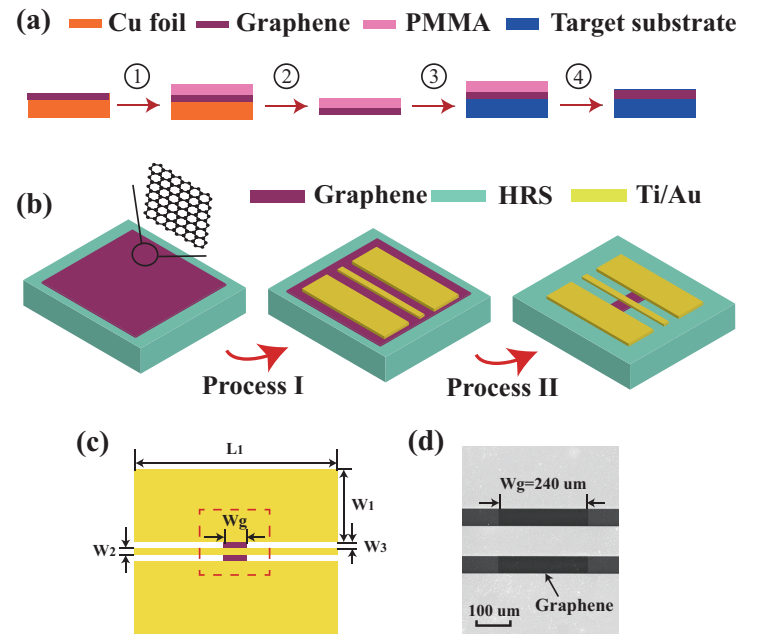

FIG. 2. (a) Graphene transfer flow charts. (b) Fabrication process flow for metal deposition and graphene patterning. (c) Layout of the graphene-integrated coplanar waveguide. (d) Scanning electron microscope (SEM) images for the dashed red-line region in (c), where the graphene flakes of $48 \times 240 \mu \mathrm{m}^{2}$ are placed. mula [42]. Partial element electrical circuit, a derived circuit-level modeling method, has also been utilized for simulations [43]. However, as mentioned in the previous section, the quality of the CVD-graphene cannot reach the ideal expectation at this stage. It hinders the realization of many theoretical simulations which employ graphene as a conductive surface. Several works, on the other hand, take the advantage of the lossy characteristic of CVD-graphene and propose the graphene-embedded microstrip attenuator [44] and tunable transmission line from experimental results [45].

\section{TUNABLE GRAPHENE-BASED ANTENNAS FROM TERAHERTZ TO MID-INFRARED}

The monolayer graphene allows to be $\mathrm{p} / \mathrm{n}$-doped under positive/negative electrostatic bias. The injection of charge carriers will shift the graphene chemical potential (Fermi level) away from the Dirac point resulting in the tunable surface conductivity. Due to the forbidden interband transitions by the Pauli blocking, the graphene monolayer reveals the metallic behavior and supports strong plasmonic effects from the terahertz to mid-infrared even to visible light regions. More importantly, the graphene monolayer with tunable chemical potential (through modulating the gated voltage) enables us to gradually engineer its surface conductivity and subsequently manipulate the graphene plasmon for realizing the tunable optical response of graphene-based antennas. In this section, we will discuss the graphene monolayer as a key element for dynamically altering the antenna characteristics.

\section{A. Manipulation of graphene absorption}

Regarding the reciprocity theorem, the graphene-based antennas are capable of receiving and transmitting electromagnetic waves. As the receiver, besides the radiation pattern, gain and impedance, the optical absorption plays an important role in converting the propagating wave into surface wave toward high responsivity and also in enhancing near-field. The modulation of the absorption of graphene-based antenna will offer in-depth insight on how to engineer the antenna characteristics.

As we know, the intrinsic value of optical absorption of undoped monolayer graphene is $2.3 \%$, which is insufficient to realize strong light-matter interaction. The graphene plasmon and photonic modes, critical coupling effects, etc, have been utilized to improve the absorption of graphene antennas. For instance, through modulating the graphene chemical potential, the log-periodic toothed antenna made by graphene has delivered the multi-resonances with highly tunable intensity and spectral location [46]. The active tunable absorption has been demonstrated in the closely packed graphene nanodisk 
arrays, in which the disk geometrical parameters, interparticle spacing and voltage-driven electrostatic doping can be optimized to achieve $30 \%$ graphene absorption with one order of magnitude enhancement as compared to that of the planar un-doped graphene monolayer [47]. In a subsequent work, Halas et. al. also demonstrated the plasmon energy and strength can be tuned in the nanostructured graphene monolayer disk array [48]. Theoretically, the critical coupling effect has been realized to attain the perfect absorption of graphene, the combined guided mode of supstrate dielectric grating and photonic bandgap of substrate Bragg grating cooperatively contribute to near-unity absorption of the graphene [49].

Besides, the integrations of graphene monolayer with various types of metallic structures also offer additional degrees of freedom for engineering the graphene absorption. Qin et al. desined the antenna designs to enhance the interaction between metallic structures and graphene layer. The graphene monolayer with $30 \%$ absorption has been achieved in an ultra-broadband spectral range from $780 \mathrm{~nm}$ to $1760 \mathrm{~nm}$ through integrating two types of the split cross antennas [50]. In addition to the absorption, the experimental results showed that the blackbody emission of graphene-based resonator can also be electrically modulated [51].

\section{B. Modulation of optical characteristics \\ 1. utilization of tunable graphene conductivity}

The electrostatic bias on graphene can gradually change its dielectric constant, which plays an important role in continuously modulating the antenna characteristics [52-54]. The remarkable modulation depth up to $90 \%$ of transmission has been demonstrated in the graphene-loaded silver ribbon antenna via turn onand off-resonance [55]. Yu and coworkers showed the large modulation of both the amplitude and phase in graphene-metal antenna. The intensity modulation ratio of 100 and phase modulation of $240^{\circ}$ have been demonstrated through dynamically tuning the graphene surface conductivity [56]. The control of the magnetic resonance of diabolo antenna by integrating graphene monolayer has been shown with the resonance tuning range up to $63 \%$ and intensity modulation up to $1460 \%$ in mid-infrared wavelength range [57]. Capasso and coworkers demonstrated the in situ control of graphene-loaded mid-infrared antenna through electrically tuning the applied gated voltage, the electrostatically gated graphene located at the antenna gap enables the change of wavelength range up to $600 \mathrm{~nm}$ with the modulation depth of the intensity more than 30\% [20]. They further demonstrated a large tuning range of $1100 \mathrm{~nm}$ ( $80 \%$ of bandwidth) at the mid-infrared region by incorporation of metal-insulator-metal waveguide design in the graphenebased antenna [58]. In another work, they studied the electrically tunable absorber composed of Fabry-Perot antenna and graphene. The gated voltage applied on the graphene enables to modulate in and out of the critical coupling condition, the modulation depth up to $100 \%$ and speed of $20 \mathrm{GHz}$ have been realized over a broadband wavelength range from the near-infrared to terahertz wavelength [59].

Yin et al. proposed the graphene-based twodimensional (2D) leaky-wave antenna allowing both the frequency tuning and beam steering in the terahertz region. The proposed structure is shown in Fig. 3(a), in which the graphene is adopted as the high impedance surface. Through dynamically controlling the gated voltage, the concomitant change of the graphene conductivity not only can effectively alter the reflection phase and resonant frequency over a wide wavelength range but also enable modulation of the radiation pattern of $2 \mathrm{D}$ leaky-wave antenna (See Fig 3(b)) [60]. The in situ control of antenna polarization is also desirable in practical applications. Jiang and Sha et al. demonstrated the graphene-based polarizer, in which the graphene monolayer is placed underneath the cross antenna that composed of two perpendicular dipole antennas (See Fig. $3(\mathrm{c}))$ [61]. The electrostatically tunable chemical potential of the graphene monolayer enables adjustment of the polarization state of the asymmetric cross antenna. The cross antenna at operational wavelength of $6 \mu \mathrm{m}$ generates a perfect circular state of the reflected beam with the axial ratio of $0.3 \mathrm{~dB}$. Moreover, the axial ratio could be significantly changed by $9 \mathrm{~dB}$ when the underneath graphene monolayer is electrostatically biased to achieve the doping level of $1 \mathrm{eV}$ (See Fig. 3(d)). The Stokes parameters evaluated within the feed gap indicate that the value of S3 (represents level of circularly polarized state) is dominant compared to that of S1 (represents the differentiate linear polarization) (See Fig. 3(e)). This confirms the reflected beam is highly circular in zero doping. The reflected beam is more linearly polarized along the $x$-direction when the chemical potential is tuned to a higher value of $1 \mathrm{eV}$. Consequently, the reflected wave has been electrically tuned from circular to near-linear states with the axial ratio varying over $8 \mathrm{~dB}$.

The modulations of the radiation pattern, directivity and efficiency of other type antennas such as the FabryPerot cavity leaky-wave antenna [62], terahertz Yagi-Uda antenna [63], the split ring resonator based Fano antenna $[64,65]$ and gold nanorod pair antenna[66] have been demonstrated by shifting the graphene chemical potential under different electrostatic biases. Besides, the graphene has also been adopted as the photoconductive source of radio-frequency antenna and load of terahertz patch antenna. Their time-domain characteristics also show interesting capabilities under dynamic modulations [67-69]. 
(a)

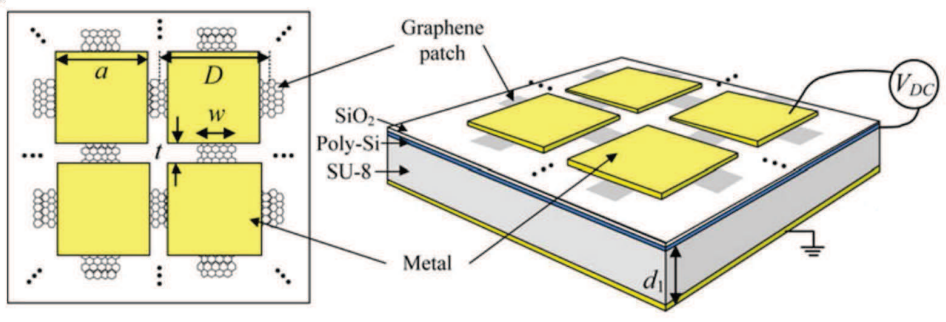

(b)

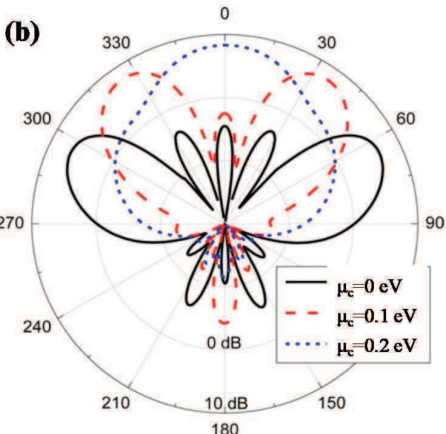

(e)

(c)

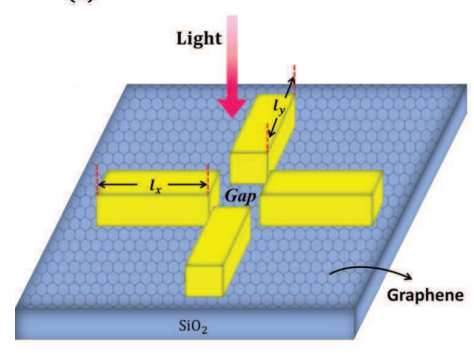

(d)

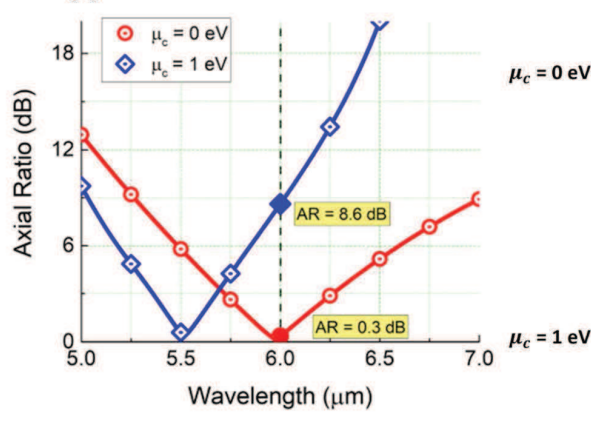

(g)

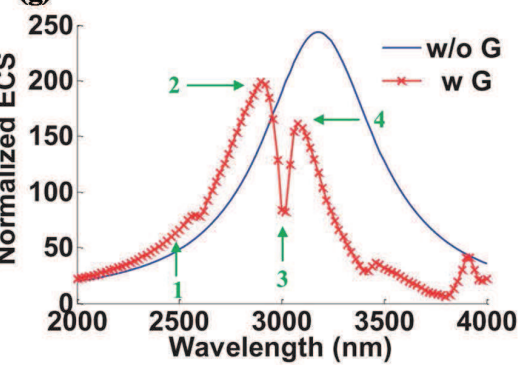

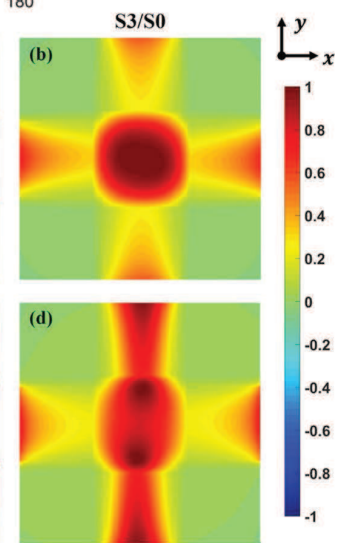

(h)

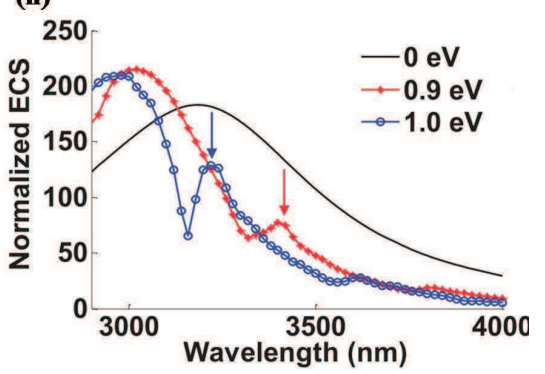

FIG. 3. (Color online) (a) Schematic of the 2D leaky-wave antenna with the graphene as a high-impedance surface, (b) E-plane radiation patterns with different chemical potential $\mu_{c}$ [60]. (c) Schematic of the unit cell of the proposed graphene-loaded electrically tunable polarizer, (d) axial ratio of the reflected beam of the cross antenna with the chemical potential of 0 eV and $1 \mathrm{eV}$, (e) Stokes parameters calculated at $20 \mathrm{~nm}$ above the polarizer top surface focusing on the polarizer feed gap [61]. (f) The near field distributions of the doped graphene monolayer $\left(\mu_{c}=1.1 \mathrm{eV}\right)$ inserted in between $4 \mathrm{~nm} \mathrm{Al}_{2} \mathrm{O}_{3}$ and $100 \mathrm{~nm} \mathrm{SiO} 2($ as shown in the inset) at the wavelength of $3000 \mathrm{~nm},(\mathrm{~g})$ the extinction cross section (ECS) of the metallic dipole antenna with and without the graphene monolayer, $(\mathrm{h})$ the extinction cross section under different chemical potentials [74].

\section{2. utilization of graphene plasmon}

The surface plasmon polaritons supported by the graphene monolayer can be dynamically controlled by the electrostatic doping level, which offer a platform to engineer its spectral resonant location and interaction with metal plasmon. The previous reports revealed that the plasmon of graphene and thin metal layer have the comparable performance [70]. The unidirectional surface plasmon polaritons launcher and the propagation of surface plasmon polaritons have been demonstrated to be electrically controllable through dynamically biasing the source/drain of graphene-based field transistor devices [71]. Yao et al. numerically investigated the plasmon-induced transparency of the graphene dipole and monopole antenna [72]. The spectral location and lineshape have been tuned by dynamically controlling the destructive interference between graphene dipole and monopole antenna. Murphy et al. unveiled the anomalously high resonant transmission of graphene-metal antenna and showed the increment of graphene mobility resulting in the enhanced resonant transmission and narrowed bandwidth [73]. Choy and coworkers have demonstrated the strong mode coupling between the graphene plasmon and metal plasmon in graphene-based dipole antenna. The near-field distribution, resonance frequency, bandwidth, radiation pattern, etc. have been dynamically tuned by the in-phase and out-of-phase couplings 
between the graphene plasmonic and metallic plasmonic [74]. The proposed structure adopted a thin metal oxide of $\mathrm{Al}_{2} \mathrm{O}_{3}$ inserted in between the graphene monolayer and metal dipole antenna for avoiding the quantum tunneling effect between the metal and graphene. The surface plasmon of graphene can be readily seen in the near field profile in Fig. 3(f). The results showed that appropriate chemical doping of graphene can induce a strong mode coupling between the graphene plasmon and metal plasmon, which results in a resonance splitting of the graphene-based dipole antenna (See Fig. 3(g)). More interestingly, the switch on/off of the mode coupling can be controlled through tuning the graphene chemical potential with electrostatically applied voltage (See Fig. 3(h)). In addition, Halas et. al. proposed the doped graphene through the injection of the plasmon-induced hot electrons, which the prominent carrier density in graphene monolayers, by varying the plasmonic antenna size, incident laser wavelength and laser power density etc., will dynamically shift the Dirac point [75]. In a subsequent work, Fang et. al. demonstrated the plasmonic hot electron generated by the gold nanoparticles can also be tunneled into graphene monolayers, which can be used as the graphene-based photodetector. The tunneling effect of the hot electrons, i.e. the characteristic of the photodetector, can be controlled by incident laser power and bias voltage between the top and bottom electrode [76].

\section{Graphene-based antenna}

\section{1. terahertz and microwave antennas}

The terahertz antennas such as the dipole antenna with the size from several to hundreds micrometers fit the small area of experimentally fabricated graphene, which is a suitable platform for extensive study of the graphenebased/modulated antennas [77-80]. For instance, the adoption of graphene nanoribbons as the terahertz antenna has been reported to modulate the mode compression factor of surface plasmon polariton and propagation [81]. Zhang and coworkers presented a dielectric grating with graphene as the leaky-wave antenna. The radiation pattern reconfigurability of the antenna has been realized by controlling the graphene plasmon through the modulation of the applied voltage on the graphene [82]. Galli et al. proposed the substrate-superstrate configured leakywave antenna with graphene as the load to tune the radiation properties [83]. Recently, Yakovlev and coworkers studied the coverage of the elliptically-shaped graphene monolayer on the two dipole antennas as the surface reactance. The mutual coupling between each dipole antenna can be modulated by the graphene chemical potential resulting in a dynamically controllable radiation pattern [84]. Chen and coworkers proposed the implementation of a circular antenna to control the beam direction, in which the graphene-metal loop acts as the reflector to dynamically manage the resonant location [85]. In the another work, the theoretical upper limits on the radiation efficiency and beam steering have been studied for the graphene-based terahertz nonreciprocal antenna [86].

Besides the linearly polarized wave, the graphene monolayer also exhibits capabilities to generate vortex waves. Jian and coworkers proposed the cross shaped antenna to generate the plasmon vortex on the coated graphene monolayer (See Fig. 4(a)). With the excitation of cross antenna under the linearly polarized light with the directions of $45^{\circ}$ and $-45^{\circ}$, the amplitude of electric field component $E_{z}$ exhibits the sharp change along the rotation direction (Fig. 4(b)). The corresponding phase profiles as shown in Fig. 4(c) readily revealed features of vortex waves. More importantly, the sign of topological charge of plasmon vortex on graphene can be controlled by the linearly polarized direction of incident light [87]. Mao and coworkers proposed the reconfigurable graphene reflectarray for the generation of vortex radio wave antenna [88]. The schematic of the reconfigurable graphene reflectarray is shown in Fig. 4(d). The dark and bright gray colors correspond to different chemical potentials. The change of the chemical potential and size of graphene monolayer enable the controllable reflection coefficients and generate the $0, \pm 1$, and \pm 2 modes of vortex beams at 1.6 terahertz. The three-dimensional radiation patterns and phase fronts of the vortex beams for the modes of $l=-1$ and $l=-2$ are shown in Figs. 4(e) and 4(f), respectively. The graphene monolayer also shows potential applications as microwave antennas [89-92]. The metalinsulator reversible transitions of graphene via switch on the electrostatic bias for low surface resistance metal and switch off for high surface resistance insulator can efficiently control the microwave antenna's radiation patterns [93].

\section{2. spatially modulated graphene for antenna applications}

The resonant optical antenna with spatial conductivity pattern has been demonstrated to launch and control the graphene plasmon [94]. The spatial modulation of the applied bias on graphene has also been studied on various antennas. Perruisseau-Carrier and coworkers proposed the concept of designing a sinusoidally modulated graphene leaky antenna by spatially controlling the gated voltage [95]. Its configuration is shown in Fig. $4(\mathrm{~g})$. The graphene surface reactance can be controlled by sinusoidally modulating the bias voltage applied on the graphene (See Fig 4(h)). It results in dynamically tuning the pointing angle and leakage rate of the antenna at a fixed frequency. The radiation pattern with different beam directions can be generated with different sampling points in one modulation period (See Fig 4(i)). Alu and coworkers proposed the graphene parallelplate waveguide loaded with gating pads and demonstrated the spatiotemporal modulation of graphene conductivity. The structure serves as plasmonic isolators and leaky-wave antennas at terahertz frequencies, which

Wang, R., Ren, XG., Yan, Z. et al. Front. Phys. (2019) 17: 13603. https://doi.org/10.1007/s11467-018-0859-y 
(a)

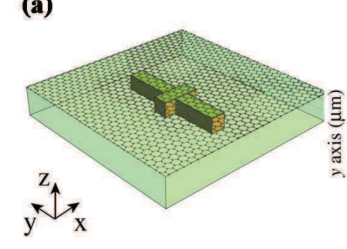

(d) $\quad \frac{\left.H_{i} I\right]_{i}^{W_{d}}}{W_{H_{d}}}$

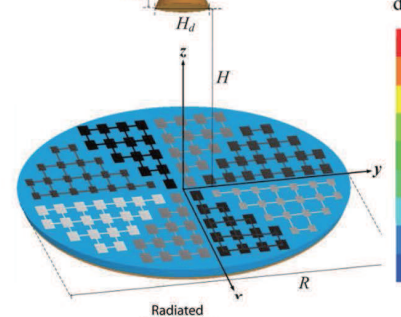

Radiated
Leaky Wave
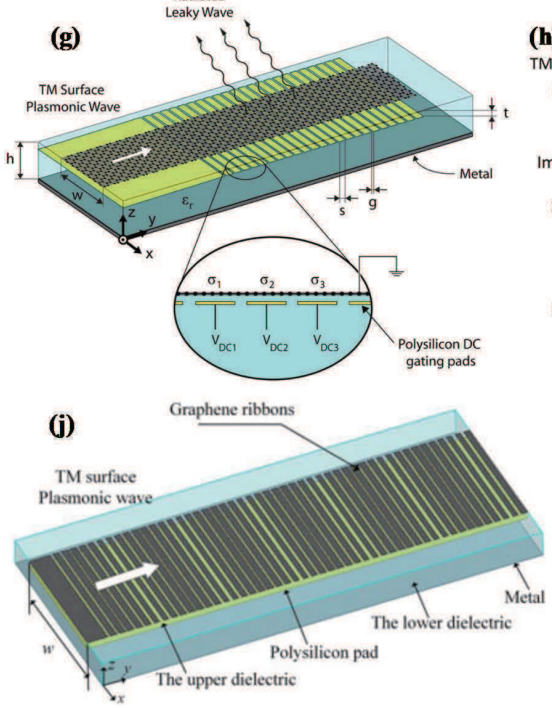

(b)

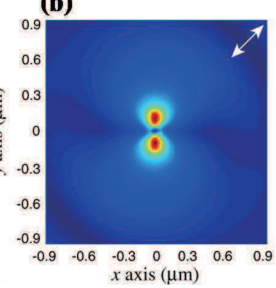

(e)

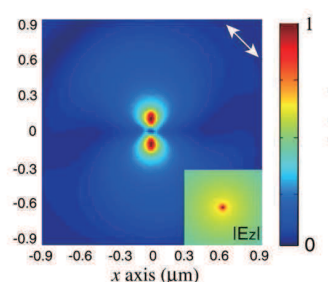

$x$ axis $(\mu \mathrm{m})$
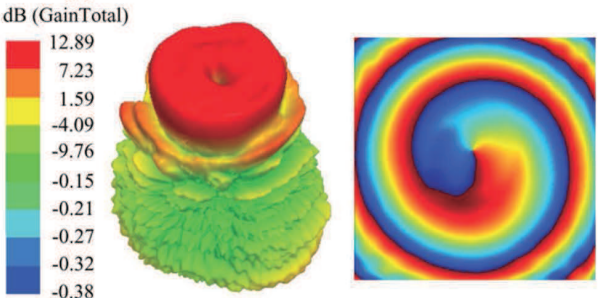

(c)
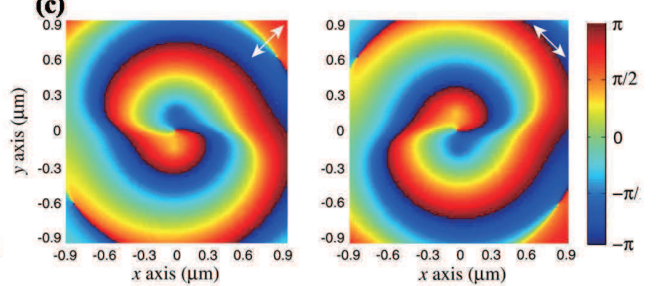

(f)

dB (GainTotal)
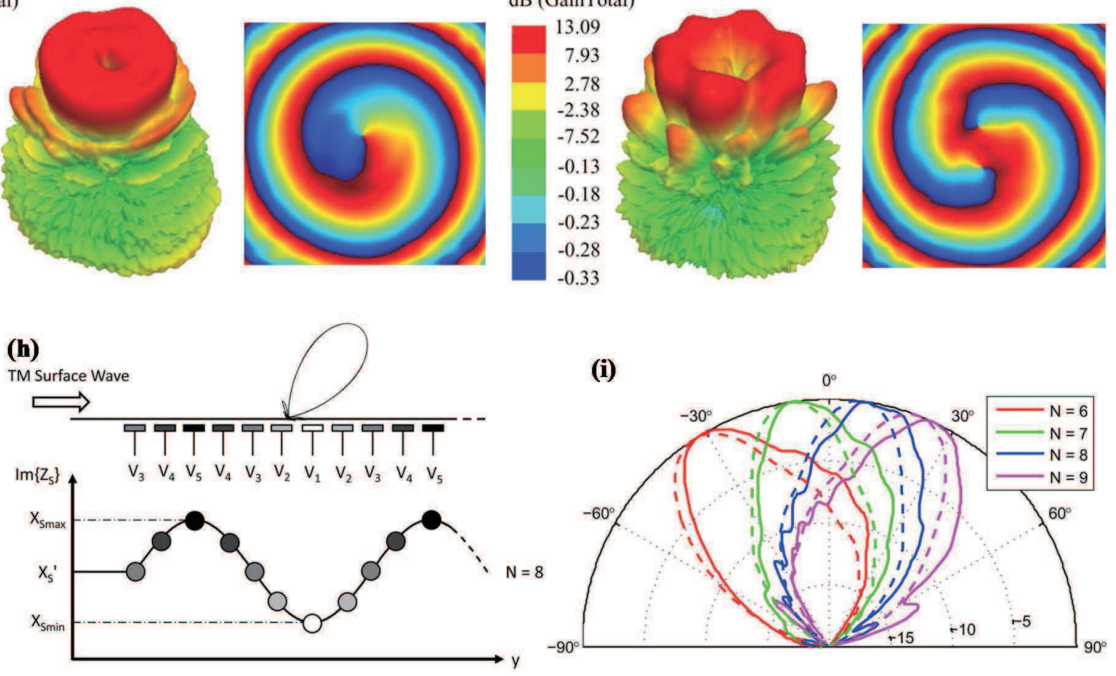

(i)
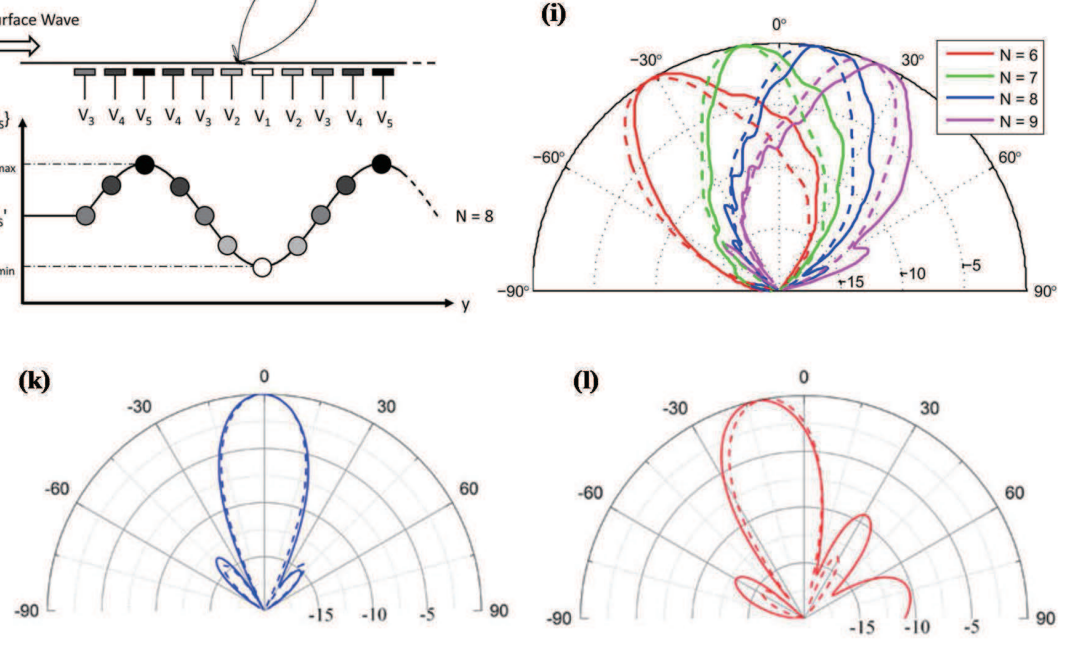

(k)

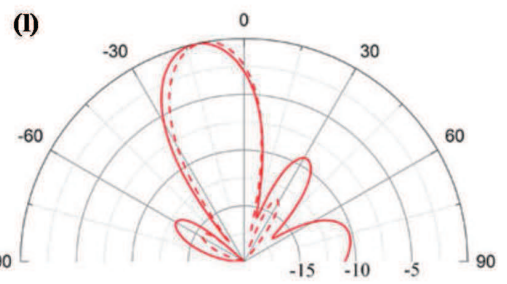

FIG. 4. (Color online) (a) Schematic view of the cross shaped metal antenna, (b) the normalized amplitude and (c) the phase profiles of electric field component $E_{z}$ excited by the cross shaped antenna under $45^{\circ}$ and $-45^{\circ}$ [87]. (d) Reconfigurable graphene reflectarray, the dark and bright gray colors correspond to different chemical potentials. The simulated threedimensional radiation patterns and phase fronts of the vortex radio waves with the modes of (e) $l=-1$ and (f) $l=-2$ [88]. (g) Schematic of the sinusoidally-modulated graphene surface reactance operating as a leaky-wave antenna. The polysilicon pads (yellow) are used to modify the graphene conductivity as a function of the applied DC voltage, (h) schematic representation of the relation between the DC bias voltage and graphene reactance, (i) radiation patterns for different values of sampling point $N$ in one modulation period [95]. (j) Schematic of the sinusoidally-modulated leaky-wave antenna with nonuniformly gapped graphene nanoribbon, the radiation patterns of the leaky antenna with the chemical potential of (k) $0.5 \mathrm{eV}$ and (l) $0.9 \mathrm{eV}$ [98].

allow independent manipulation of graphene properties in both space and time toward the nonreciprocal device [96]. They further extended the concept to design the infrared beamformer through modulating the graphene with elastic vibrations based on flexural waves. A fast on-off switching of infrared emission and dynamic tuning of radiation pattern, beam angle and frequency of operation have been realized [97]. Mao and coworkers demonstrated the dynamical tuning of beam scanning of a sinusoidally-modulated graphene leaky-wave antenna with only one biasing voltage [98]. They have realized the leaky-wave antenna through designing the graphene nanoribbon with the nonuniform gap size, whose configuration is shown in Fig 4(j). The nonuniformly gapped graphene ribbons can achieve a sinusoidally-modulated surface reactance to support the leaky-wave operation. With the change of chemical potential from $0.5 \mathrm{eV}$ to $0.9 \mathrm{eV}$, the radiation beam can be turned from pointing angle of $0^{\circ}$ to $15^{\circ}$. (See Figs. $4(\mathrm{k})$ and $4(\mathrm{l})$ ) 


\section{3. graphene-based antenna for photodetection}

In the terahertz and visible light regions, the rectifying antenna is used to convert electromagnetic energy into direct current electricity that has promising applications in photodetector and wireless communication systems [99102]. The introduction of graphene for modulating the responsivity of photodetector and rectenna also have received intensive attentions. Capasso and coworkers have demonstrated a metallic antenna with simultaneously improved light absorption and photocarrier collection in graphene-based detectors. The metallic antenna serves as the light trapping structure for substantial absorption enhancement and the electrode for efficient carrier collection. The resultant responsivity of the metallic antenna-assisted graphene detector has been enhanced over 200 times [103]. Halas and coworkers also proposed a graphene antenna as the photodetector, in which the antenna is sandwiched between two graphene monolayers. The plasmon of the antenna will benefit the direct excitation of graphene electrons and also induce hot electrons that are subsequently transferred to graphene. They cooperatively enhance the photocurrent with 8 times [104]. For the wireless communication, various graphene-based patch antennas with low profiles and small sizes have also been comprehensively studied. The adopted graphene monolayer offers the possibilities to actively control the directivity, and bandwidth of the patch antenna [105110]. The graphene monolayer has also been reported to fabricate the radio frequency identification tag [111-113], and realize the multiple-input multiple-output antenna system with tunable beamwidth and radiation direction $[114,115]$.

\section{GRAPHENE PLASMONIC MODULATORS AT TERAHERTZ AND INFRARED}

Modulating the light intensity, phase, and polarization plays a critical role in most optical imaging and communication systems. Compared to modulators operating at telecommunication wavelengths that are predominantly used for fiber communications, terahertz or infrared spatial light modulators are uniquely targeted to environment/health monitoring, navigation, and surveillance through scattering foggy or dusty environments.

Due to its large conductivity swing, graphene has attracted lots of attention as a material for terahertz modulators [116-119]. Since 2010s, there have been many reports for a large modulation depth and broadband operation in the terahertz regime by actively controlling the carrier concentration of a graphene layer and via the integration of graphene with metamaterials [13, 116-131]. In 2012, Sensale-Rodriguez et al. [132] developed a broadband graphene terahertz electro-absorption modulator. By applying a voltage between a graphene layer and a silicon substrate, in a large-area field-effect transistor-like configuration, the Fermi level in the graphene film can be well controlled. Although the modulation depth achieved using this technique is remarkable, owing to the one-atom thickness of graphene as the active material, the overall device performance is still far away from application expectations. However, there are several ways to enlarge the modulation depth. One of them is to utilize the reflection mode structures [134]. Integrating graphene into such structures can boost the absorption in graphene due to electric field enhancement when the distance between graphene and a reflector (e.g. a metallic layer coated on the back of the substrate to which graphene is transferred) is matched to an odd multiple of a quarter wavelength [134]. The experimental reflectance as a function of bias voltage shows that at the frequency at which the substrate thickness is matched to an odd multiple of a quarter wavelength, i.e. 0.62 terahertz maximum reflectance modulation is attained, and $>64 \%$ amplitude modulation with $2 \mathrm{~dB}$ insertion loss is possible in this structure. However, owing to the characteristics of its geometry, the response of this structure is narrow band.

On the other hand, terahertz modulation is also attainable in graphene plasmonic structures. In this regard, Ju et al. [136] proposed a new device design composed of stripes of graphene. By changing the carrier density in graphene, it is possible to shift the geometric plasmonic resonance of the device. One step further is to integrate graphene with metamaterials. It takes advantage of the electric field enhancement due to the metamaterial in order to boost the light-matter interaction in graphene [139-150]. In 2012, Lee et al. [149] reported a graphene metamaterial modulators with a very large modulation depth $(86 \%)$, but at the expenses of a large insertion loss $(>10 \mathrm{~dB})$. As depicted schematically in Fig. 5, two extraordinary transmission electrodes are placed on both sides of this layered structure so as to gate the active terahertz graphene metamaterial (Fig. 5(a)). And the metaatoms composed of a hexagonal metallic frame or asymmetric double split rings (aDSRs) exhibiting Fano-like resonance are periodically arranged (Fig. 5(b)) [149]. An optical micrograph of the fabricated graphene metamaterial attached to a printed circuit board $(\mathrm{PCB})$ is shown in Fig. 5(c). As shown in Fig. 5(d), the fabricated active graphene metamaterial is large-area $\left(15 \times 15 \mathrm{~mm}^{2}\right)$, fully flexible and free-standing without the thick base substrate that is generally required for semiconductorbased terahertz modulators [149].

From the experimental results of the phase shift versus applied bias, a $65^{\circ}$ phase shift is achieved at a frequency of $0.9 \mathrm{THz}$. Here we would like to note that in these structures there is always a tradeoff between the modulation depth, insertion loss and the bandwidth of modulation. Later in 2014, a low-bias terahertz modulator based on the integration of split-ring resonator and a graphene layer was further proposed by Degl'Innocenti [151]. The modulation in a broad spectral range $(2.2-3.1$ terahertz) with a modulation depth of up to $18 \%$ was achieved. In 2012, a metamaterial terahertz modulator based on periodic gold slit arrays with graphene as an active load of 
a
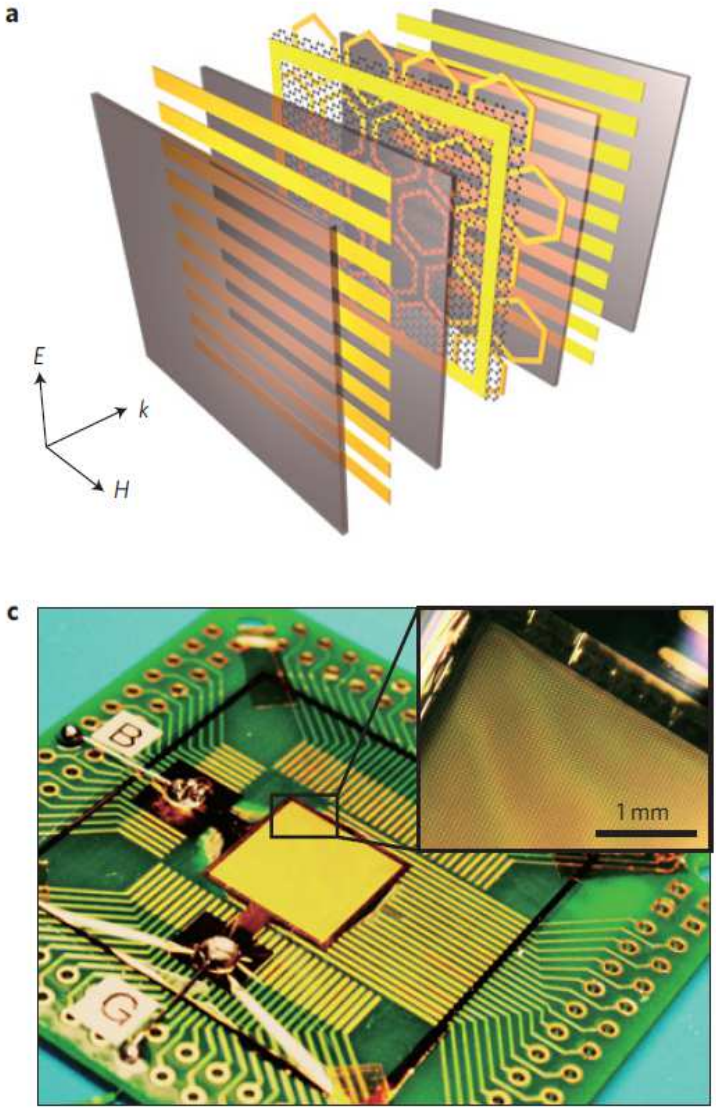

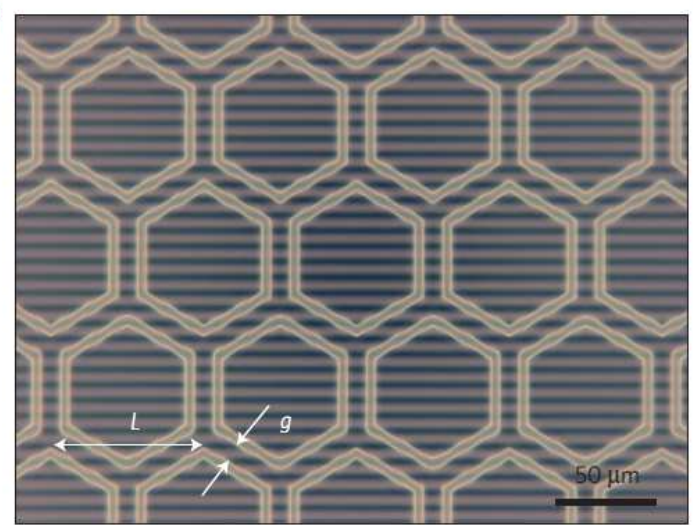

d

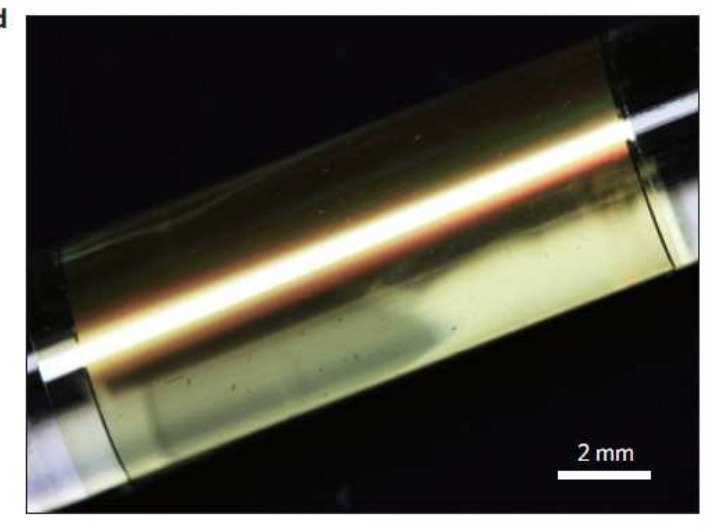

FIG. 5. (Color online) Schematic view and device images of gate-controlled active graphene metamaterials. (a) Schematic rendering of a gate-controlled active graphene metamaterial composed of a SLG deposited on a layer of hexagonal metallic meta-atoms (a unit cell of $L=60 \mu \mathrm{m}$ and $g=5 \mu \mathrm{m}$ ) and top/bottom EOT electrodes embedded in a dielectric material of 4 thickness. Metallic patterns and dielectric materials are represented by yellow and grey, respectively. The polarization of the incident terahertz wave is perpendicular to the line electrode, as indicated by the arrows. (b) Optical micrograph of the fabricated gate-controlled active graphene metamaterial without the top electrode. (c) Fully integrated gate-controlled active graphene metamaterial attached to a drilled PCB for THz-TDS measurement (B, connected to bottom EOT electrode; G, connected to graphene layer). Inset: magnified view of the gate-controlled active graphene metamaterial. (d) Optical image of the fabricated large-area metamaterial wound round a glass rod, showing its high degree of flexibility. Source: Reprinted with permission from Ref. [149]. Copyright from 2012 Nature Publishing Group.

tunable conductivity was proposed in [152] and further experimentally demonstrated in 2015 by Shi et al. in [153]. A sketch of this structure is schematically depicted in Fig. 6(a). The field enhancement in the graphene layer is attained via the coherent radiation of the enhanced inplane local-field in the slits, thus preserving the broadband terahertz modulation of bare graphene in the hybrid device. Thus this structure is capable of efficiently coupling terahertz radiation while exhibiting tunability of the terahertz transmission over a broad frequency range as shown in Fig. 6(b) and Fig. 6(d). The schematic side view of the ion-gel gated device is shown as the inset of Fig. 6(c) [153]. Under this scheme strong terahertz absorption and a large local field enhancement exist, leading to a modulation depth of $90 \%$. It is also possible to improve the modulation depth in these structures by increasing the number of graphene layers. It should be noted that as the number of layers increases, the insertion loss also increases. Furthermore, in 2015, Wu et al. [154] reported a broadband terahertz modulator based on graphene/ionic liquid/graphene sandwich structures, in which the obtained modulation depth reached up to $99 \%$ in the frequency range $0.1-2.5$ terahertz.

One interesting approach towards high-performance graphene modulators is via the utilization of hybrid systems. By optically pumping the structure and properly engineering the device, it is possible to obtain significant modulation depth $[155,156]$. A graphene terahertz modulator based on an optical pumping approach was demonstrated by Wen et al. in [156], which was based 

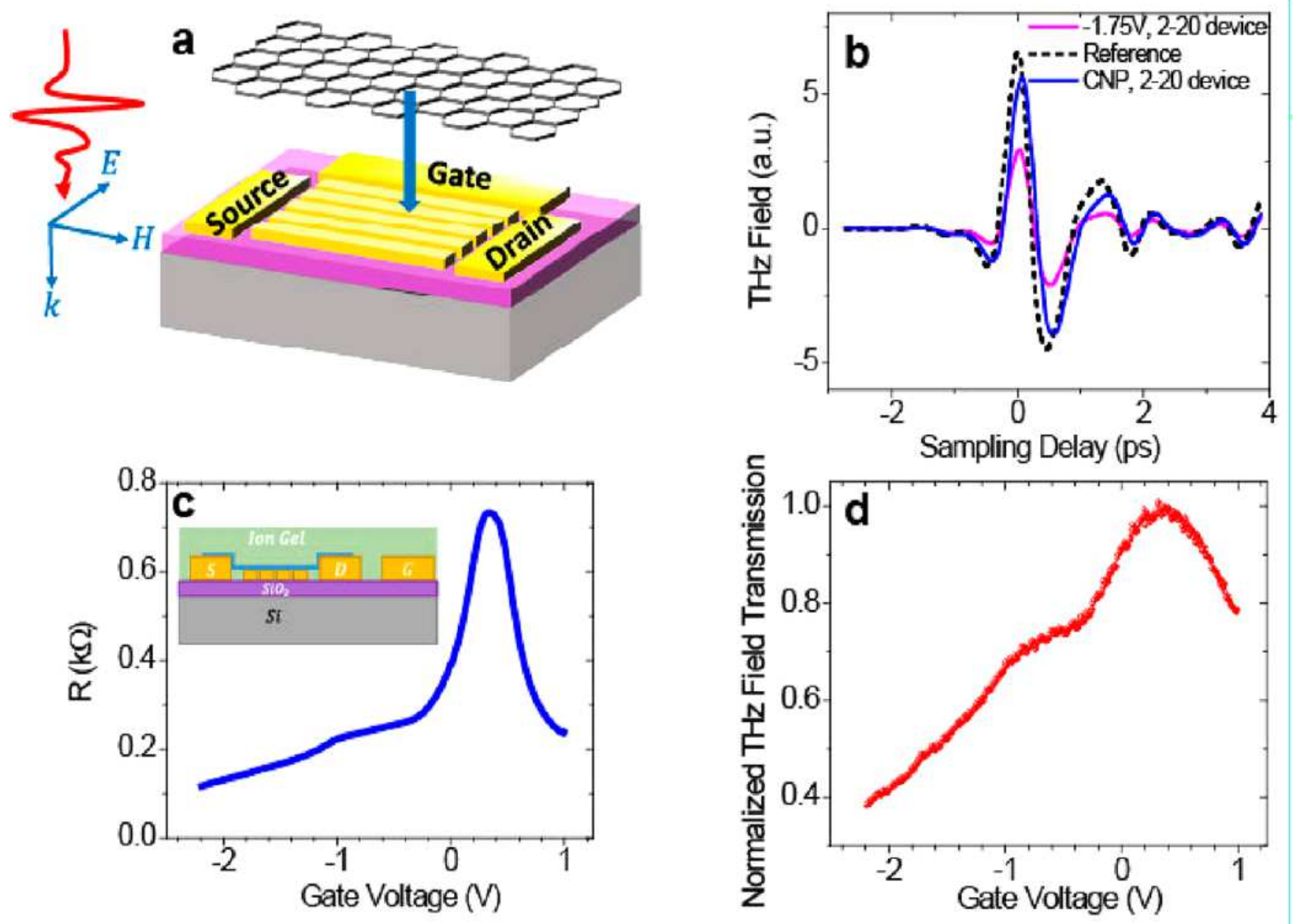

FIG. 6. (Color online) Terahertz modulation by the graphene/metasurface hybrid device. (a) Schematic representation of the experiment setup for measurement as well as the hybrid device configuration. We fabricate the hybrid device by transferring a single layer graphene on top of a gold slit device of width $2 \mu \mathrm{m}$ and period $20 \mu \mathrm{m}$. The incoming terahertz wave is polarized perpendicular to the slit orientation. (b) Transmitted terahertz waveforms for a reference sample (dashed black trace), the hybrid device with graphene at charge neutrality point (CNP, solid blue trace), and the hybrid device with graphene at gate voltage $-1.75 \mathrm{~V}$ (solid magenta trace). (c) Resistance measured for this hybrid device shows charge neutrality point at the gate voltage of $0.33 \mathrm{~V}$. The side view of a schematic representation of our ion-gel gated device is shown in the inset, where the blue line represents graphene. (d) Simultaneously measured terahertz field transmission of this hybrid device (normalized to the transmission when graphene is at charge neutrality point). Source: Reprinted with permission from Ref. [153]. Copyright from 2015 American Chemistry Society.

on single-layer graphene on germanium. The modulator was driven optically with a low power $1.55 \mu \mathrm{m}$ laser, in which the main mechanism to enable the terahertz modulation is the third-order nonlinear effect $[157,158]$ in the optical conductivity of the graphene. The modulator shows wide-band modulation in the range $0.25-1$ terahertz with a modulation depth up to $94 \%$. In 2015 , Liang et al. [160] demonstrated that $>94 \%$ modulation depth can be attained via the monolithic integration of a surface-emitting terahertz quantum cascade laser featuring a concentric circular grating with modulation speeds up to $110 \mathrm{MHz}$. The improvement in performance and speed is achieved as a result of the small area of the device and the stronger interaction of the terahertz radiation with the graphene layer, due to monolithic inte- gration of modulator and source. Moreover, a graphenesilicon hybrid device was experimentally demonstrated by employing optical excitation and direct-current bias together as the actuation mechanism [161]. This new graphene-silicon configuration device with a 'diode' behavior is actuated under simultaneous continuous wave photoexcitation and direct-current bias voltage, which exhibited a relatively large modulation depth up to $83 \%$ at a low gate bias voltage of $4 \mathrm{~V}$.

\section{GRAPHENE DETECTORS AT TERAHERTZ}

Although several room temperature field effect transistors have been shown capable of detecting terahertz 
waves with very fast response and high responsivity in the $0.3-3$ terahertz range based on InAs nanowire field-effect transistors [162-164], graphene plasmonics as one exciting frontier offers lies in harvesting low energy photons for robust photodetection at terahertz at room temperature, which can potentially overcome the limitations of conventional semiconductor terahertz devices. It oughts to be noted that, since the thermal diffusion of hot carriers instead of lattice heating is responsible for generating the current, photo-thermoelectric graphene detectors can achieve fast frequency response. It could be generalizable to terahertz frequencies, where carriers in graphene have an even stronger response [164]. Conventional terahertz and sub-terahertz detection systems based on incoherent (pyroelectric, Golay cell, Si bolometers) or coherent (heterodyne mixers) approaches are either very slow ( $100 \mathrm{~Hz}$ modulation frequency) or require deep cryogenic cooling, while those exploiting fast nonlinear electronics (Schottky diodes) show a significant drop of performance above 1 terahertz [163].Traditional detectors based on interband transitions (e.g., mercury cadmium telluride) require cryogenic cooling to suppress darkcurrent noise, especially when operating at long wavelength infrared wavelengths. Moreover, device miniaturization and their heterogeneous integration with silicon or germanium platforms remain challenging. Uncooled thermal detectors such as thermopiles or bolometers usually possess large device footprints, low responsivities and slow speeds due to thermal time constants being in the millisecond range. These drawbacks have significantly impeded their applications in thermal imaging and free space communications.

Recently, it has been generally recognized that graphene can pave the way to robust and cheap terahertz detectors operating at room temperature based on the Dyakonov and Shur scheme [162, 169]. Graphene has a very high carrier mobility, even at room temperature [1]. Furthermore, it supports plasma waves that are weakly damped in high-quality samples [7, 19]. Thus, singlelayer and bilayer graphene field-effect transistor plasmabased photodetectors could outperform other terahertz detector technologies. In the last couple of years, various graphene based structures have been proposed i.e. single-layer or bilayer graphene-insulator-graphene heterostructure tunnel field-effect transistor [170-173], lateral single-layer or bilayer graphene field-effect transistor [174], vertical cascade multiple graphene layer structures [175], etc., for resonant detections. Moreover, some interesting works demonstrate that such graphene-insulatorgraphene heterostructure tunnel field-effect transistor structures can show strong resonant terahertz detections, compared to ungated tunnel field-effect transistor structures, and interesting readers can refer to Refs. [162, 164, 169, 176].

Graphene has also been proposed for the broadband terahertz detection by employing a simple top-gate antenna-coupled configuration for the excitation of overdamped plasma waves in the channel of a graphene field- effect transistor [65]. Several further studies on similar split-bow tie antenna have also been reported to improve the coupling and thereby responsivity at 0.34 terahertz and 0.6 terahertz [177-179]. On the other hand, some interesting results were also obtained for detections at 1.63 and 3.11 terahertz using graphene field-effect transistors, whose drain and source contact leads worked as antennas for the incoming terahertz radiation [180]. Moreover, several photodection devices have also been demonstrated in the mid-infrared and far infrared regimes [181, 182]. In Ref. [182], the bilayer graphene was used as the bilayer graphene small electron heat capacity; and weak electron-phonon coupling/interaction creates a bottleneck in the heat path, thus leading to a temperature dependent resistance induced by the large change in electron temperature, which can be then converted to a detectable electrical signal. The developed detector exhibits a voltage responsivity about $2 \times 10^{5} \mathrm{VW}^{-1}$ and an electrical noise-equivalent power about $33 \mathrm{fW} \mathrm{Hz}^{-1 / 2}$ at $5 \mathrm{~K}$ with a very high intrinsic speed of the device $(>1$ $\mathrm{GHz}$ at $10 \mathrm{~K}$ ). Besides, room temperature terahertz detection using the photothermoelectric effect in graphene has also been shown experimentally $[183,184]$.

Besides, the graphene monolayers have also been widely explored as other functional devices such as the transistors, sensors, memorizers, etc. The graphenebased transistors have shown great promising to reach a smaller channel length and higher speed, which offer the opportunities to outperform the existing devices and become an option for post-silicon electronics [185-187]. Recently, Sahakian et. al. demonstrated the cascaded spintronic logic with graphene nanoribbon based transistors. The proposed computing systems permit terahertz operation speed and two orders of magnitude decrement in power-delay product as compared to the current cuttingedge microprocessors [188]. The unique characteristics of the graphene monolayers are also of critical use in the next-generation memorizers due to its superiorities in bit density, energy efficiency and long data retention time [189]. The graphene monolayers have been applied in the resistive random memory, phase-change memory, spintransfer-torque magnetic random access memory and ferroelectric random access memory etc., in which the graphene can be used as the memory electrode due to its flexibility and transparency, and interfacial engineering layer due to the block of atomic diffusion, reduction of power consumption etc. [190-194]. The graphenebased non-volatile memory devices has been systematically summarized in previous works $[189,195]$. In addition, the graphene field effect transistor can also be used as the smart sensor, in which the advantages of graphene monolayers such as the large surface-to-volume ratio, uniquely tunable optical properties, excellent carrier mobility, and exceptional electrical and thermal properties, are suitable for sensing $[187,196]$. There are several works on the graphene-based strain sensor, electrochemical sensor, biosensor and electrical sensor (including the temperature sensing, photodetector, etc.), in which the 
graphene reveal great potential to achieve the low detection limit and high sensitivity [196-199]. Notably, the graphene-based sensors have the low influence on the surrounding environment as compared to the metalbased sensors. Nalwa et. al. have detailed reviewed the flexible graphene-based wearable gas and chemical sensors in the detection of various hazard gases, toxic heavy ions, and volatile organic compounds etc. [200]. In addition, the decorations of the graphene monolayers with metal nanostructures permit the large enhancement of the chemical detection capabilities [201], the intensified surface-enhanced Raman scattering has been reported with metal nanoparticle/graphene/metal film system [202-204]. Consequently, the graphene monolayers have already shown great potential in functional devices and promised in future practical applications.

\section{CONCLUSION}

Graphene represents an emerging frontier that brings together multidisciplines of physics, material science, electronics, photonics and terahertz plasmonics. It has uncovered exciting prospects in these fields, where novel technologies and solutions are still being developed. From the device and system viewpoint, graphene is a two-dimensional layered material with an intrinsically passivated surface. It is thus amenable to monolithic integration with conventional silicon-based benchmarks as well as other important functional materials, which makes graphene remarkably advantageous over bulk materials whose heterogeneous integration with other materials usually induces defects and deep level trapping centers at the interface.

Presently graphene has been suffering some demanding challenges that must be overcome before making an impact on industry-standard device applications. Among many others, the high quality synthesis of large-scale graphene is of paramount importance and will have the most positive impact on this field. Specifically, the improvements in graphene's crystal quality (i.e., carrier mobility) can significantly prolong the lifetime of graphene plasmons, which is strongly correlated with various device performance metrics, such as the light-matter in- teraction strength, absorbance, sensitivity,phase shifts, spectral resolution, modulation efficiency, detectivity, and so forth.

Moreover, there remains a most important requirement to gain a detailed and fundamental physical understanding of the hot carrier dynamics and transports in graphene since they are indispensable for efficiently developing high-performance graphene-based informatic functional devices. Furthermore, the nonlocal, nonlinear and quantum effects existing in deep subwavelength graphene nanostructures deserve to be intensively investigated in future as well, which will open up a new platform for quantum information, communication, and measurement. The light-matter interaction will occur at both the atomic/molecular level and single-photon level. Additionally, the graphene based multi-functional on-chip devices operating at microwave, terahertz, and visible light frequencies are expected to be invented.

In summary, the various physical and chemical properties of graphene make it well suited for integrated, multifunctional and compact devices and systems, yet its potential has not been fully reached. In this review, we offered a brief on graphene based transmission lines, antennas, modulators, and detectors covering from microwave frequency band to terahertz or infrared band. We believe that future progresses on graphene-based functional devices and microsystems will lead to proliferation in both fundamental investigations and applied technological products for imaging, communication, environmental monitoring, medical diagnostics, spectroscopic studies etc.

\section{ACKNOWLEDGMENTS}

This research work is supported by the National Key R\&D Program of China (Grant No. 2016YFE0204200), the Natural Science Foundation of China (61701003) and Natural Science Research Foundation of Anhui Province (No. 1808085QF179). This work is also supported by the Research Grants Council of Hong Kong GRF 17210815, NSFC 61271158, HKU Seed Fund 201711159228, AOARD FA2386-17-1-0010, and Hong Kong UGC AoE/P-04/08.
[1] A. H. Castro Neto, F. Guinea, N. M. R. Peres, K. S. Novoselov, A. K. Geim, The electronic properties of graphene, Rev Mod Phys 81, 109-162 (2009).

[2] A. K. Geim, Graphene: Status and Prospects, Science 324, 1530-1534 (2009).

[3] M. J. Allen, V. C. Tung, R. B. Kaner, Honeycomb Carbon: A Review of Graphene, Chem Rev 110, 132-145 (2010).

[4] P. Avouris, Graphene: Electronic and Photonic Properties and Devices, Nano Lett, 10,4285-4294 (2010).

[5] F. Bonaccorso, Z. Sun, T. Hasan, A. C. Ferrari,
Graphene photonics and optoelectronics, Nat Photonics 4, 611-622 (2010).

[6] Q. L. Bao, K. P. Loh, Graphene Photonics, Plasmonics, and Broadband Optoelectronic Devices, ACS Nano 6, 3677-3694 (2012).

[7] A. N. Grigorenko, M. Polini, K. S. Novoselov, Graphene plasmonics, Nat Photonics 6, 749-758 (2012).

[8] K. S. Novoselov, V. I. Fal'ko, L. Colombo, P. R. Gellert, M. G. Schwab, K. Kim, A roadmap for graphene, Nature 490, 192-200 (2012).

[9] P. Tassin, T. Koschny, M. Kafesaki, C. M. Soukoulis, 
A comparison of graphene, superconductors and metals as conductors for metamaterials and plasmonics, Nat Photonics 6, 259-264 (2012).

[10] F. J. G. de Abajo, Graphene Nanophotonics, Science 339, 917-918 (2013).

[11] F. J. G. de Abajo, Graphene Plasmonics: Challenges and Opportunities, ACS Photonics 1, 135-152 (2014).

[12] T. Low, P. Avouris, Graphene Plasmonics for Terahertz to Mid-Infrared Applications, ACS Nano 8, 1086-1101 (2014).

[13] T. Otsuji, V. Popov, V. Ryzhii, Active graphene plasmonics for terahertz device applications, J Phys D Appl Phys 47, 094006 (2014).

[14] T. Stauber, Plasmonics in Dirac systems: from graphene to topological insulators, J Phys-Condens Mat 26, 123201 (2014).

[15] N. K. Emani, A. V. Kildishev, V. M. Shalaev, A. Boltasseva, Graphene: A Dynamic Platform for Electrical Control of Plasmonic Resonance, Nanophotonics-Berlin, 4 (2015) 214-223.

[16] S. S. Xiao, X. L. Zhu, B. H. Li, N. A. Mortensen, Graphene-plasmon polaritons: From fundamental properties to potential applications, Front Phys-Beijing 11, 117801 (2016).

[17] S. Y. Huang, C. Y. Song, G. W. Zhang, H. G. Yan, Graphene plasmonics: physics and potential applications, Nanophotonics-Berlin 6, 1191-1204 (2017).

[18] V. P. Gusynin, S. G. Sharapov, J. P. Carbotte, Magneto-optical conductivity in graphene, J. Phys.: Condens. Matter 19, 026222 (2007).

[19] M. Jablan, H. Buljan, M. Soljačić, Plasmonics in graphene at infrared frequencies, Phys. Rev. B 80, 245435 (2009).

[20] Y. Yao, M. A. Kats, P. Genevet, N. Yu, Y. Song, J. Kong and F. Capasso, Broad electrical tuning of grapheneloaded plasmonic antennas, Nano Lett. 13(3), 1257-1264 (2013).

[21] V. Nayyeri, M. Soleimani, O. M. Ramahi, Modeling graphene in the finite-difference time-domain method using a surface boundary condition, IEEE Trans. Antennas Propag. 61(8), 4176-4182 (2013).

[22] P. Li, L. J. Jiang, H. Bagci, A resistive boundary condition enhanced DGTD scheme for the transient analysis of graphene, IEEE Trans. Antennas Propag. 63(7), 3065-3076 (2015).

[23] P. Li, L. J. Jiang, Modeling of magnetized graphene from microwave to $\mathrm{THz}$ range by DGTD with a scalar $\mathrm{RBC}$ and an ADE, IEEE Trans. Antennas Propag. 63(10), 4458-4467 (2015).

[24] Y Shao, J. J. Yang, M. Huang, A Review of Computational Electromagnetic Methods for Graphene Modeling, Int. J. Antenn. Propag. 2016, 7478621 (2016).

[25] K. S. Novoselov, D. Jiang, F. Schedin, T. J. Booth, V. V. Khotkevich, S. V. Morozov, A. K. Geim, Twodimensional atomic crystals, Proc. Natl. Acad. Sci. 102(30), 10451-10453 (2005).

[26] P. Neugebauer, M. Orlita, C. Faugeras, A. L. Barra, M. Potemski, How perfect can graphene be?, Phys. Rev. Lett. 103(13), 136403 (2009).

[27] D. C. Elias, R. V. Gorbachev, A. S. Mayorov, S. V. Morozov, A. A. Zhukov, P. Blake, A. K. Geim, Dirac cones reshaped by interaction effects in suspended graphene, Nat. Phys. 7(9), 701 (2011).

[28] C. Berger, Z. Song, X. Li, X. Wu, N. Brown, C. Naud,
E. H. Conrad, Electronic confinement and coherence in patterned epitaxial graphene, Science 312(5777), 11911196 (2006).

[29] K. V. Emtsev, A. Bostwick, K. Horn, J. Jobst, G. L. Kellogg, L. Ley, E. Rotenberg, Towards wafer-size graphene layers by atmospheric pressure graphitization of silicon carbide, Nat. Mater. 8(3), 203 (2009).

[30] W. A. De Heer, C. Berger, M. Ruan, M. Sprinkle, X. Li, Y. Hu, E. Conrad, Large area and structured epitaxial graphene produced by confinement controlled sublimation of silicon carbide, Proc. Natl. Acad. Sci. 108(41), 16900-16905 (2011).

[31] J. Hass, J. E. Millán-Otoya, P. N. First, E. H. Conrad, Interface structure of epitaxial graphene grown on $4 \mathrm{H}-$ SiC (0001), Phys. Rev. B 78(20), 205424 (2008).

[32] Y. M. Lin, C. Dimitrakopoulos, K. A. Jenkins, D. B. Farmer, H. Y. Chiu, A. Grill, P. Avouris, 100-GHz transistors from wafer-scale epitaxial graphene, Science 327(5966), 662-662 (2010).

[33] R. F. Davis, G. Kelner, M. Shur, J. W. Palmour, J. A. Edmond, Thin film deposition and microelectronic and optoelectronic device fabrication and characterization in monocrystalline alpha and beta silicon carbide, Proc. IEEE 79(5), 677-701 (1991).

[34] X. Li, W. Cai, J. An, S. Kim, J. Nah, D. Yang, S. K. Banerjee, Large-area synthesis of high-quality and uniform graphene films on copper foils, Science 324(5932), 1312-1314 (2009).

[35] N. Petrone, C. R. Dean, I. Meric, A. M. van Der Zande, P. Y. Huang, L. Wang, J. Hone, Chemical vapor deposition-derived graphene with electrical performance of exfoliated graphene, Nano Lett. 12(6), 27512756 (2012)

[36] X. Li, C. W. Magnuson, A. Venugopal, J. An, J. W. Suk, B. Han, L. Fu, Graphene films with large domain size by a two-step chemical vapor deposition process, Nano Lett. 10(11), 4328-4334 (2010).

[37] C. R. Dean, A. F. Young, I. Meric, C. Lee, L. Wang, S. Sorgenfrei, J. Hone, Boron nitride substrates for highquality graphene electronics, Nat. Nanotech. 5(10), 722 (2010).

[38] R. Wang, S. Raju, M. Chan, L. J. Jiang, Low frequency behavior of CVD graphene from DC to $40 \mathrm{GHz}$, Prog. Electromagn. Res. 71, 1-7 (2017).

[39] M. Tamagnone, J. S. Gomez-Diaz, J. R. Mosig, J. Perruisseau-Carrier, Analysis and design of terahertz antennas based on plasmonic resonant graphene sheets, J. Appl. Phys. 112(11), 114915 (2012).

[40] Z. Chang, L. S. Wu, M. Tang, Y. P. Zhang, J. F. Mao, Generation of $\mathrm{THz}$ wave with orbital angular momentum by graphene patch reflectarray, Advanced Materials and Processes for $\mathrm{RF}$ and $\mathrm{THz}$ Applications (IMWS-AMP), 2015 IEEE MTT-S International Microwave Workshop, pp. 1-3 (2015).

[41] Y. L. Xu, X. C. Wei, E. P. Li, Three-dimensional tunable frequency selective surface based on vertical graphene micro-ribbons, J. Electromagnet. Wave 29(16), 2130-2138 (2015).

[42] G. W. Hanson, Dyadic Green's functions and guided surface waves for a surface conductivity model of graphene, J. Appl. Phys. 103(6), 064302 (2008).

[43] Y. S. Cao, L. J. Jiang, A. E. Ruehli, An equivalent circuit model for graphene-based terahertz antenna using the PEEC method, IEEE Trans. Antennas Propag. 
64(4), 1385-1393 (2016).

[44] L. Pierantoni, D. Mencarelli, M. Bozzi, R. Moro, S. Moscato, L. Perregrini, S. Bellucci, Broadband microwave attenuator based on few layer graphene flakes, IEEE Trans. Microw. Theory 63(8), 2491-2497 (2015).

[45] R. Wang, L. J. Jiang, Electrically tunable behavior of graphene on high-resistivity silicon substrate, Antennas and Propagation and USNC/URSI National Radio Science Meeting, 2017 IEEE International Symposium, pp. 1031-1032 (2017).

[46] J. Yang, F. Kong and K. Li, Broad Tunable Nanoantenna Based on Graphene Log-Periodic Toothed Structure, Plasmonics 11(4), 981 (2015).

[47] Z. Fang, Y. Wang, A. E. Schlather, Z. Liu, P. M. Ajayan, F. J. de Abajo, P. Nordlander, X. Zhu and N. J. Halas, Active tunable absorption enhancement with graphene nanodisk arrays, Nano Lett. 14(1), 299 (2014).

[48] Z. Y. Fang, Y. M. Wang, Z. Liu, A. Schlather, P. M. Ajayan, F. H. L. Koppens, P. Nordlander and N. J. Halas, Plasmon-Induced Doping of Graphene. Acs Nano 6 (11), 10222 (2012).

[49] J. Wu, Tunable ultranarrow spectrum selective absorption in a graphene monolayer at terahertz frequency, $J$. Phys. D-Appl. Phys. 49(21), 215108 (2016).

[50] F. Xiong, J. Zhang, Z. Zhu, X. Yuan and S. Qin, Ultrabroadband, More than One Order Absorption Enhancement in Graphene with Plasmonic Light Trapping, Sci. Rep. 5, 16998 (2015).

[51] V. W. Brar, M. C. Sherrott, M. S. Jang, S. Kim, L. Kim, M. Choi, L. A. Sweatlock and H. A. Atwater, Electronic modulation of infrared radiation in graphene plasmonic resonators, Nat. Commun. 6, 7032 (2015).

[52] B. Mehta and M. E. Zaghloul,Tuning the Scattering Response of the Optical Nano Antennas Using Graphene, IEEE Photonics J. 6(1), 4800208 (2014).

[53] D. Sikdar, W. Zhu, W. Cheng and M. Premaratne, Substrate-Mediated Broadband Tunability in Plasmonic Resonances of Metal Nanoantennas on Finite High-Permittivity Dielectric Substrate, Plasmonics 10(6), 1663 (2015).

[54] A. Locatelli, G. E. Town and C. De Angelis, GrapheneBased Terahertz Waveguide Modulators, IEEE Trans. Terahertz Sci. Technol. 5(3), 351 (2015).

[55] R. Yu, V. Pruneri and F. J. Garcia de Abajo, Active modulation of visible light with graphene-loaded ultrathin metal plasmonic antennas, Sci. Rep. 6, 32144 (2016).

[56] Z. Li and N. Yu, Modulation of mid-infrared light using graphene-metal plasmonic antennas, Appl. Phys. Lett. 102(13), 131108 (2013).

[57] N. Yi, Z. Liu, S. Sun, Q. Song and S. Xiao, Mid-infrared tunable magnetic response in graphene-based diabolo nanoantennas., Carbon 94, 501 (2015).

[58] Y. Yao, M. A. Kats, R. Shankar, Y. Song, J. Kong, M. Loncar and F. Capasso, Wide wavelength tuning of optical antennas on graphene with nanosecond response time, Nano Lett. 14(1), 214 (2014).

[59] Y. Yao, R. Shankar, M. A. Kats, Y. Song, J. Kong, M. Loncar and F. Capasso, Electrically tunable metasurface perfect absorbers for ultrathin mid-infrared optical modulators, Nano Lett. 14(11), 6526 (2014).

[60] X. C. Wang, W. S. Zhao, J. Hu and W. Y. Yin, Reconfigurable Terahertz Leaky-Wave Antenna Using Graphene-Based High-Impedance Surface, Ieee T. Nan- otechnol. 14(1), 62 (2015).

[61] Y. Qin, X. Y. Z. Xiong, W. E. I. Sha and L. J. Jiang, Electrically tunable polarizer based on graphene-loaded plasmonic cross antenna, J. Phys. Condens. Matter. 30(14), 144007 (2018).

[62] W. Fuscaldo, P. Burghignoli, P. Baccarelli and A. Galli, Graphene Fabry-Perot Cavity Leaky-Wave Antennas: Plasmonic Versus Nonplasmonic Solutions, Ieee T. Antenn. Propag. 65(4), 1651 (2017).

[63] Y. Wu, M. Qu, L. Jiao, Y. Liu and Z. Ghassemlooy, Graphene-based Yagi-Uda antenna with reconfigurable radiation patterns, AIP Adv. 6(6), 065308 (2016).

[64] A. Hosseinbeig, A. Pirooj and F. B. Zarrabi, A reconfigurable subwavelength plasmonic fano nano-antenna based on split ring resonator, J. Magn. Magn. Mater. 423, 203 (2017).

[65] F. B. Zarrabi, M. Mohaghegh, M. Bazgir and A. S. Arezoomand, Graphene-Gold Nano-ring antenna for Dualresonance optical application, Opt. Mater. 51, 98 (2016).

[66] Z. Dong, C. Sun, J. Si and X. Deng, A tunable plasmonic nano-antenna based on metal-graphene doublenanorods, Laser Phys. Lett. 15 (5), 056202 (2018).

[67] A. Cabellos-Aparicio, I. Llatser, E. Alarcon, A. Hsu and T. Palacios, Use of Terahertz Photoconductive Sources to Characterize Tunable Graphene RF Plasmonic Antennas, Ieee T. Nanotechnol. 14(2), 390 (2015).

[68] M. M. Seyedsharbaty and R. A. Sadeghzadeh, Antenna gain enhancement by using metamaterial radome at $\mathrm{THz}$ band with reconfigurable characteristics based on graphene load, Opt. Quant. Electron. 49(6), 221 (2017).

[69] S. Abadal, I. Llatser, A. Mestres, H. Lee, E. Alarcon and A. Cabellos-Aparicio, Time-Domain Analysis of Graphene-Based Miniaturized Antennas for UltraShort-Range Impulse Radio Communications, Ieee. T. Commun. 63(4), 1470 (2015).

[70] X. He, P. Gao and W. Shi, A further comparison of graphene and thin metal layers for plasmonics, Nanoscale 8(19), 10388 (2016).

[71] Y. Bao, S. Zu, Y. Zhang and Z. Fang, Active Control of Graphene-Based Unidirectional Surface Plasmon Launcher, ACS Photonics 2(8), 1135 (2015).

[72] X. L. Zhao, C. Yuan, L. Zhu and J. Q. Yao, Graphenebased tunable terahertz plasmon-induced transparency metamaterial, Nanoscale 8(33), 15273 (2016).

[73] M. M. Jadidi, A. B. Sushkov, R. L. Myers-Ward, A. K. Boyd, K. M. Daniels, D. K. Gaskill, M. S. Fuhrer, H. D. Drew and T. E. Murphy, Tunable Terahertz Hybrid Metal-Graphene Plasmons, Nano Lett. 15(10), 7099 (2015).

[74] X. G. Ren, W. E. I. Sha and W. C. H. Choy, Tuning optical responses of metallic dipole nanoantenna using graphene, Opt. Express 21(26), 31824 (2013).

[75] Z. Y. Fang, S. Thongrattanasiri, A. Schlather, Z. Liu, L. L. Ma, Y. M. Wang, P. M. Ajayan, P. Nordlander, N. J. Halas and F. J. G. de Abajo, Gated Tunability and Hybridization of Localized Plasmons in Nanostructured Graphene. Acs Nano 7 (3), 2388 (2013).

[76] B. Du, L. Lin, W. Liu, S. Zu, Y. Yu, Z. Li, Y. Kang, H. Peng, X. Zhu and Z. Fang, Plasmonic hot electron tunneling photodetection in vertical Au-graphene hybrid nanostructures. Laser \& Photonics Reviews 11 (1), (2017).

[77] R. Filter, M. Farhat, M. Steglich, R. Alaee, C. Rockstuhl and F. Lederer, Tunable graphene antennas for 
selective enhancement of THz-emission, Opt. Express 21(3), 3737 (2013).

[78] T. Zhou, Z. Cheng, H. Zhang, M. Le Berre, L. Militaru and F. Calmon, Miniaturized tunable terahertz antenna based on graphene Microw. Opt. Techn. Let. 56(8), 1792 (2014).

[79] M. Dragoman, M. Aldrigo, A. Dinescu, D. Dragoman and A. Costanzo, Towards a terahertz direct receiver based on graphene up to $10 \mathrm{THz}, J$. Appl. Phys. 115(4), 044307 (2014).

[80] D. Correas-Serrano, J. S. Gomez-Diaz, A. Alu and A. Alvarez-Melcon, Electrically and Magnetically Biased Graphene-Based Cylindrical Waveguides: Analysis and Applications as Reconfigurable Antennas, IEEE Trans. Terahertz Sci. Technol. 5(6), 951 (2015).

[81] J. M. Jornet and I. F. Akyildiz, Graphene-based Plasmonic Nano-Antenna for Terahertz Band Communication in Nanonetworks, Ieee. J. Sel. Area. Comm. 31(12), 685 (2013).

[82] J. Li, M. He, C. Wu and C. Zhang, Radiation Pattern Reconfigurable Graphene Leaky-Wave Antenna at Terahertz Band Based on Dielectric Grating Structure, Ieee Antennas Wirel. Propag. Lett. 16, 1771 (2017).

[83] W. Fuscaldo, P. Burghignoli, P. Baccarelli and A. Galli, A Reconfigurable Substrate?Superstrate GrapheneBased Leaky-Wave THz Antenna, Ieee Antennas Wirel. Propag. Lett. 15, 1545 (2016).

[84] G. Moreno, H. Mehrpour Bernety and A. B. Yakovlev, Reduction of Mutual Coupling Between Strip Dipole Antennas at Terahertz Frequencies With an Elliptically Shaped Graphene Monolayer, Ieee Antennas Wirel. Propag. Lett. 15, 1533 (2016).

[85] X. H. Cheng, Y. Yao, S. W. Qu, Y. L. Wu, J. S. Yu and X. D. Chen, Circular beam-reconfigurable antenna base on graphene-metal hybrid, Electron Lett. 52(7), 494 (2016).

[86] M. Tamagnone and J. R. Mosig, Theoretical Limits on the Efficiency of Reconfigurable and Nonreciprocal Graphene Antennas, Ieee Antennas Wirel. Propag. Lett. 15, 1549 (2016).

[87] B. Zhu, G. Ren, Y. Gao, B. Wu, Y. Lian and S. Jian, Creation of Graphene Plasmons Vortex via Cross Shape Nanoantennas Under Linearly Polarized Incidence, Plasmonics 12(3), 863 (2016).

[88] Z. Chang, B. You, L.-S. Wu, M. Tang, Y.-P. Zhang and J.-F. Mao, A Reconfigurable Graphene Reflectarray for Generation of Vortex $\mathrm{THz}$ Waves, Ieee Antennas Wirel. Propag. Lett. 15, 1537 (2016).

[89] S. Kosuga, R. Suga, O. Hashimoto and S. Koh, Graphene-based optically transparent dipole antenna, Appl. Phys. Lett. 110(23), 233102 (2017).

[90] T. T. Tung, S. J. Chen, C. Fumeaux and D. Losic, Scalable realization of conductive graphene films for high-efficiency microwave antennas, J. Mater. Chem. C 4(45), 10620 (2016)

[91] M. Dragoman, D. Neculoiu, A.-C. Bunea, G. Deligeorgis, M. Aldrigo, D. Vasilache, A. Dinescu, G. Konstantinidis, D. Mencarelli, L. Pierantoni and M. Modreanu, A tunable microwave slot antenna based on graphene, Appl. Phys. Lett. 106(15), 153101 (2015).

[92] C. Nunez Alvarez, R. Cheung and J. S. Thompson, Performance Analysis of Hybrid Metal?Graphene Frequency Reconfigurable Antennas in the Microwave Regime, Ieee. T. Antenn. Propag. 65(4), 1558 (2017).
[93] M. Aldrigo, M. Dragoman and D. Dragoman, Smart antennas based on graphene, J. Appl. Phys. 116(11), 114302 (2014).

[94] P. Alonso-Gonzalez, A. Y. Nikitin, F. Golmar, A. Centeno, A. Pesquera, S. Velez, J. Chen, G. Navickaite, F. Koppens, A. Zurutuza, F. Casanova, L. E. Hueso and R. Hillenbrand, Controlling graphene plasmons with resonant metal antennas and spatial conductivity patterns, Science 344(6190), 1369 (2014).

[95] M. Esquius-Morote, J. S. Gomez-Diaz and J. Perruisseau-Carrier, Sinusoidally Modulated Graphene Leaky-Wave Antenna for Electronic Beamscanning at THz, IEEE Trans. Terahertz Sci. Technol. 4(1), 116 (2014).

[96] D. Correas-Serrano, J. S. Gomez-Diaz, D. L. Sounas, Y. Hadad, A. Alvarez-Melcon and A. Alu, Nonreciprocal Graphene Devices and Antennas Based on Spatiotemporal Modulation, Ieee Antennas Wirel. Propag. Lett. 15, 1529 (2016).

[97] P. Y. Chen, M. Farhat, A. N. Askarpour, M. Tymchenko and A. Alu, Infrared beam-steering using acoustically modulated surface plasmons over a graphene monolayer, J. Opt. 16(9), 094008 (2014).

[98] Y. Cheng, L.-S. Wu, M. Tang, Y.-P. Zhang and J.-F. Mao, A Sinusoidally-Modulated Leaky-Wave Antenna With Gapped Graphene Ribbons, Ieee Antennas Wirel. Propag. Lett. 16, 3000 (2017).

[99] Z. Zhu, S. Joshi, S. Grover and G. Moddel, Graphene geometric diodes for terahertz rectennas, J. Phys. DAppl. Phys. 46(18), 185101 (2013).

[100] C. Chakraborty, R. Beams, K. M. Goodfellow, G. W. Wicks, L. Novotny and A. Nick Vamivakas, Optical antenna enhanced graphene photodetector, Appl. Phys. Lett. 105(24), 241114 (2014).

[101] X. Huang, T. Leng, M. Zhu, X. Zhang, J. Chen, K. Chang, M. Aqeeli, A. K. Geim, K. S. Novoselov and Z. $\mathrm{Hu}$, Highly Flexible and Conductive Printed Graphene for Wireless Wearable Communications Applications, Sci. Rep. 5, 18298 (2015).

[102] M. Mittendorff, S. Winnerl, J. Kamann, J. Eroms, D. Weiss, H. Schneider and M. Helm, Ultrafast graphenebased broadband $\mathrm{THz}$ detector, Appl. Phys. Lett. 103(2), 021113 (2013).

[103] Y. Yao, R. Shankar, P. Rauter, Y. Song, J. Kong, M. Loncar and F. Capasso, High-responsivity mid-infrared graphene detectors with antenna-enhanced photocarrier generation and collection, Nano Lett. 14(7), 3749 (2014).

[104] Z. Fang, Z. Liu, Y. Wang, P. M. Ajayan, P. Nordlander and N. J. Halas, Graphene-antenna sandwich photodetector, Nano Lett. 12(7), 3808 (2012).

[105] S. Anand, D. Sriram Kumar, R. J. Wu and M. Chavali, Graphene nanoribbon based terahertz antenna on polyimide substrate, Optik 125(19), 5546 (2014).

[106] A. S. Thampy, M. S. Darak and S. K. Dhamodharan, Analysis of graphene based optically transparent patch antenna for terahertz communications, Physica E 66, 67 (2015).

[107] S. A. Naghdehforushha and G. Moradi, High directivity plasmonic graphene-based patch array antennas with tunable $\mathrm{THz}$ band communications, Optik 168, 440 (2018).

[108] R. Bala and A. Marwaha, Characterization of graphene for performance enhancement of patch antenna in $\mathrm{THz}$

Wang, R., Ren, XG., Yan, Z. et al. Front. Phys. (2019) 116 13603. https://doi.org/10.1007/s11467-018-0859-y 
region, Optik 127(4), 2089 (2016).

[109] Y. Dong, P. Liu, D. Yu, G. Li and F. Tao, DualBand Reconfigurable Terahertz Patch Antenna With Graphene-Stack-Based Backing Cavity, Ieee Antennas Wirel. Propag. Lett. 15, 1541 (2016).

[110] R. Bala and A. Marwaha, Development of computational model for tunable characteristics of graphene based triangular patch antenna in $\mathrm{THz}$ regime, J. Comput. Electron. 15(1), 222 (2015).

[111] P. Kopyt, B. Salski, M. Olszewska-Placha, D. Janczak, M. Sloma, T. Kurkus, M. Jakubowska and W. Gwarek, Graphene-Based Dipole Antenna for a UHF RFID Tag, Ieee. T. Antenn. Propag. 64(7), 2862 (2016).

[112] M. Akbari, M. W. A. Khan, M. Hasani, T. Bjorninen, L. Sydanheimo and L. Ukkonen, Fabrication and Characterization of Graphene Antenna for Low-Cost and Environmentally Friendly RFID Tags, Ieee Antennas Wirel. Propag. Lett. 15, 1569 (2016).

[113] X. Huang, T. Leng, X. Zhang, J. C. Chen, K. H. Chang, A. K. Geim, K. S. Novoselov and Z. Hu, Binderfree highly conductive graphene laminate for low cost printed radio frequency applications, Appl. Phys. Lett. 106(20), 203105 (2015).

[114] Z. Xu, X. Dong and J. Bornemann, Design of a Reconfigurable MIMO System for $\mathrm{THz}$ Communications Based on Graphene Antennas, IEEE Trans. Terahertz Sci. Technol. 4(5), 609 (2014).

[115] S. Abadal, E. Alarcon, A. Cabellos-Aparicio, M. C. Lemme and M. Nemirovsky, Graphene-Enabled Wireless Communication for Massive Multicore Architectures, IEEE Commun. Mag. 51(11), 137 (2013).

[116] B. Sensale-Rodriguez, T. Fang, R. Yan, M. M. Kelly, D. Jena, L. Liu, H. G. Xing, Unique prospects for graphene-based terahertz modulators, Appl. Phys. Lett. 99, 113104 (2011).

[117] X. He, S. Kim, Tunable terahertz graphene metamaterials, Carbon 86, 237 (2015).

[118] A. Tredicucci, M. S. Vitiello, Device concepts for graphene-based terahertz photonics, IEEE J. Sel. Top. Quantum Electron. 20(1), 8500109 (2014).

[119] M. Tamagnone, A. Fallahi, J. R. Mosig, J. PerruisseauCarrier, Fundamental limits and near-optimal design of graphene modulators and non-reciprocal devices, Nat. Photonics 8, 556 (2014).

[120] Y. Chen, H. Huang, D. Akinwande, A. Alu, Graphenebased plasmonic platform for reconfigurable terahertz nanodevices, ACS Photonics 1(8), 647-654 (2014).

[121] M. Rahm, J.-S. Li, W. J. Padilla, THz wave modulators: A brief review on different modulation techniques, $J$. Infrared, Millim., Terahertz Waves 34(1), 1-27 (2013).

[122] P.-Y. Chen, A. Alu, Terahertz metamaterial devices based on graphene nanostructures, IEEE Trans. Terahertz Sci. Technol. 3(6), 748-756 (2013).

[123] H. Tanoto, L. Ding, J. H. Teng, Tunable terahertz metamaterials, Terahertz Sci. Technol. 6(1), 1-25 (2013).

[124] S. H. Lee, H.-D. Kim, H. J. Choi, B. Kang, Y. R. Cho, B. Min, Broadband modulation of terahertz waves with non-resonant graphene meta-devices, IEEE Trans. Terahertz Sci. Technol. 3(6), 764-771 (2013).

[125] Y. Zhou, X. Xu, H. Fan, Z. Ren, J. Bai, L. Wang, Tunable magnetoplasmons for efficient terahertz modulator and isolator by gated monolayer graphene, Phys. Chem. Chem. Phys. 15, 5084-5090 (2013).

[126] A. Andryieuski, A. V. Lavrinenko, Graphene metamate- rials based tunable terahertz absorber: effective surface conductivity approach, Opt. Express 21 (7), 9144-9155 (2013).

[127] M. Amin, M. Farhat, H. Bagci, A dynamically reconfigurable fano metamaterial through graphene tuning for switching and sensing applications, Sci. Rep. 3, 2105 (2013).

[128] B. Vasić, M.M. Jakovljević, G. Isić, R. Gajić, Tunable metamaterials based on split ring resonators and doped graphene, Appl. Phys. Lett. 103011102 (2013).

[129] K. Yang, S. Liu, S. Arezoomandan, A. Nahata, B. Sensale-Rodriguez, Graphene-based tunable metamaterial terahertz filters, Appl. Phys. Lett. 105(9), 093105 (2014).

[130] Y. Bludov, V. N. MR Peres, M. I. Vasilevskiy, Unusual reflection of electromagnetic radiation from a stack of graphene layers at oblique incidence, J. Opt. 15(11), 11404 (2013).

[131] J.-T. Liu, N.-H. Liu, L. Wang, X.-H. Deng, F.-H. Su, Gate-tunable nearly total absorption in graphene with resonant metal back reflector, Europhys. Lett. 104(5), 57002 (2013).

[132] B. Sensale-Rodriguez, R. Yan, M. M. Kelly, T. Fang, K. Tahy, W. S. Hwang, D. Jena, L. Liu, H. G. Xing, Broadband graphene terahertz modulators enabled by intraband transitions, Nature Commun. 3, 780 (2012).

[133] I. Maeng, S. Lim, S. J. Chae, Y. H. Lee, H. Choi, J.H. Son, Gate-controlled Nonlinear conductivity of dirac fermion in graphene field-effect transistors measured by terahertz time-domain spectroscopy, Nano Lett. 12(2), 551-555 (2012).

[134] B. Sensale-Rodriguez, R. Yan, S. Rafique, M. Zhu, W. Li, X. Liang, D. Gundlach, V. Protasenko, M. M. Kelly, D. Jena, L. Liu, H. G. Xing, Extraordinary control of terahertz beam reflectance in graphene electroabsorption modulators, Nano Lett. 12(9), 4518-4522 (2012).

[135] B. Sensale-Rodriguez, S. Rafique, R. Yan, M. Zhu, V. Protasenko, D. Jena, L. Liu, H. G. Xing, Terahertz imaging employing graphene modulator arrays, Opt. Express 21(2), 2324-2330 (2013).

[136] L. Ju, B. Geng, J. Horng, C. Girit, M. Martin, Z. Hao, H. A. Bechtel, X. Liang, A. Zettl, Y. R. Shen, F. Wang, Graphene plasmonics for tunable terahertz metamaterials, Nature Nanotechnol. 6, 630-634 (2011).

[137] B. Sensale-Rodriguez, R. Yan, M. Zhu, D. Jena, L. Liu, H. G. Xing, Efficient terahertz electro-absorption modulation employing graphene plasmonic structures, Appl. Phys. Lett. 101(26), 261115 (2012).

[138] X.-J. He, T.-Y. Li, L. Wang, J.-M. Wang, J.-X. Jiang, G.-H. Yang, F.-Y. Meng, Q. Wu, Electrically tunable terahertz wave modulator based on complementary metamaterial and graphene, J. Appl. Phys. 115, 17B903 (2014).

[139] Y. Zhang, Y. Feng, B. Zhu, J. Zhao, T. Jiang, Graphene based tunable metamaterial absorber and polarization modulation in terahertz frequency, Opt. Express 22(19), 22743-22752 (2014).

[140] J. Ding, B. Arigong, H. Ren, M. Zhou, J. Shao, M. Lu, Y. Chai, Y. Lin, H. Zhang, Tuneable complementary metamaterial structures based on graphene for single and multiple transparency windows, Sci. Rep. 4, 6128 (2014).

[141] Z. Wang, M. Zhou, X. Lin, H. Liu, H. Wang, F. Yu, S.

Wang, R., Ren, XG., Yan, Z. et al. Front. Phys. (2019) 114: 13603. https://doi.org/10.1007/s11467-018-0859-y 
Lin, E. Li, H. Chen, A circuit method to integrate metamaterial and graphene in absorber design, Opt. Commun. 329, 76-80 (2014).

[142] B. Grześkiewicz, A. Sierakowski, J. Marczewski, N. Palka, E. Wolarz, Polarization-insensitive metamaterial absorber of selective response in terahertz frequency range, J. Opt. 16(10), 105104 (2014).

[143] L. Wang, X. Chen, A. Yu, Y. Zhang, J. Ding, W. Lu, Highly sensitive and wideband tunable terahertz response of plasma waves based on graphene field effect transistors, Sci. Rep. 4, 5470 (2014).

[144] N. Born, M. Scheller, M. Koch, J. V. Moloney, Cavity enhanced terahertz modulation, Appl. Phys. Lett. 104, 103508 (2014).

[145] B. Wang, X. Zhang, K.P. Loh, J. Teng, Tunable broadband transmission and phase modulation of light through graphene multilayers, J. Appl. Phys. 115, 213102 (2014).

[146] D. Ansell, I. P. Radko, Z. Han, F. J. Rodriguez, S. I. Bozhevolnyi, and A. N. Grigorenko, Hybrid graphene plasmonic waveguide modulators, Nature Communications 6, 8846 (2015).

[147] Q. Mao, Q.-Y. Wen, W. Tian, T.-L. Wen, Z. Chen, Q.H. Yang, H.-W. Zhang, High-speed and broadband terahertz wave modulators based on large-area graphene field-effect transistors, Opt. Lett. 39(19), 5649-5652 (2014).

[148] X. He, H. Lu, Graphene-supported tunable extraordinary transmission, Nanotechnology 25(32), 325201 (2014).

[149] S. H. Lee, M. Choi, T.-T. Kim, S. Lee, M. Liu, X. Yin, H. K. Choi, S. S. Lee, C.-G. Choi, S.-Y. Choi, X. Zhang, B. Min, Switching terahertz waves with gate-controlled active graphene metamaterials, Nature Mater. 11, 936941 (2012).

[150] R. Yan, B. Sensale-Rodriguez, L. Liu, D. Jena, H. G. Xing, A new class of tunable metamaterial terahertz modulators, Opt. Express 20(27), 28664-28671 (2012).

[151] R. Degl-Innocenti, D. S. Jessop, Y. D. Shah, J. Sibik, J. A. Zeitler, P. R. Kidambi, S. Hofmann, H. E. Beere, D. A. Ritchie, Low-bias terahertz amplitude modulator based on split-ring resonators and graphene, ACS Nano 8(3) 2548-2554 (2014).

[152] A. Novitsky, A. M. Ivinskaya, M. Zalkovskij, R. Malureanu, P. Uhd Jepsen, A. V. Lavrinenko, Non-resonant terahertz field enhancement in periodically arranged nanoslits, J. Appl. Phys. 112, 074318 (2012).

[153] S.-F. Shi, B. Zeng, H.-L. Han, X. Hong, H.-Z. Tsai, H. S. Jung, A. Zettl, M. F. Crommie, F. Wang, Optimizing broadband terahertz modulation with hybrid graphene/metasurface structures, Nano Lett. 15, 372377 (2015).

[154] Y. Wu, C. Laovorakiat, X. Qiu, J. Liu, P. Deorani, K. Banerjee, J. Son, Y. Chen, E. E. M. Chia, H. Yang, Graphene terahertz modulators by ionic liquid gating, Adv. Mater. 27(11), 1874-1879 (2015).

[155] P. Weis, J. L. Garcia-Pomar, M. Höh, B. Reinhard, A. Brodyanski, M. Rahm, Spectrally wide-band terahertz wave modulator based on optically tuned graphene, ACS Nano 6(10), 9118-9124 (2012).

[156] Q.-Y. Wen, W. Tian, Q. Mao, Z. Chen, W.-W. Liu, Q.H. Yang, M. Sanderson, H.-W. Zhang, Graphene based all-optical spatial terahertz modulator, Sci. Rep. 4, 7409 (2014).
[157] S. A. Mikhailov, Non-linear graphene optics for terahertz applications, Microelectron. J. 40, 712-715 (2009).

[158] K. Yang, S. Arezoomandan, B. Sensale-Rodriguez, The linear and nonlinear thz properties of graphene, Terahertz Sci. Technol. 6(4), 223-233 (2016).

[159] I. Khromova, A. Andryieuski, A. Lavrinenko, Ultrasensitive terahertz/infrared waveguide modulators based on multilayer graphene metamaterials, Laser Photonics Rev. 8(6), 916-923 (2014)

[160] G. Liang, X. Hu, X. Yu, Y. Shen, L. H. Li, A. G. Davies, E. H. Linfield, H. K. Liang, Y. Zhang, S. F. Yu, Q. J. Wang, Integrated terahertz graphene modulator with 100 modulation depth, ACS Photonics 2(11), 1559-1566 (2015).

[161] Q. Li, Z. Tian, X. Zhang, R. Singh, L. Du, J. Gu, W. Zhang, Active graphene-silicon hybrid diode for terahertz waves, Nature Commun. 6, 7082 (2015).

[162] A. Tredicucci, M. S. Vitiello, Device concepts for graphene-based terahertz photonics, IEEE J. Sel. Top. Quantum Electron. 20, 8500109 (2007).

[163] F. Sizov, A. Rogalski, THz detectors, Prog. Quantum Electron. 34, 278-347 (2010).

[164] N. Zhang; R.L.Song; M. Hu; G.C. Shan; C.T. Wang; J. Yang A Low-Loss Design of Bandpass Filter at the Terahertz Band, IEEE Microwave and Wireless Components Letters 28 (7), 573 - 575 (2018).

[165] A. Pitanti, D. Coquillat, D. Ercolani, L. Sorba, F. Teppe, W. Knap, G. De Simoni, F. Beltram, A. Tredicucci, M. S. Vitiello, Terahetz detection by heterostructed inas/insb nanowire based field effect transistors, Appl. Phys. Lett. 101, 141103 (2012).

[166] W. Knap, M. Dyakonov, D. Coquillat, F. Teppe, N. Dyakonova, J. Lausakowski, K. Karpierz, M. Sakowicz, G. Valusis, D. Seliuta, I. Kasalynas, A. El Fatimy, Y. M. Meziani, T. Otsuji, Field effect transistors for terahertz detection: Physics and first imaging applications, J. Infrared Millim. Terahertz Waves 30, 1319-1337 (2009).

[167] E. Ojefors, U. R. Pfeiffer, A. Lisauskas, H. G. Roskos, A $0.65 \mathrm{THz}$ focal-plane array in a quarter-micron CMOS process technology, IEEE J. Solid-State Circuits 44, 1968-1976 (2009).

[168] M. B. Lundeberg, Y. Gao, A. Woessner, C. Tan, P. Alonso-González, K. Watanabe, T. Taniguchi, J. Hone, R. Hillenbrand, F. H. L. Koppens, Thermoelectric detection and imaging of propagating graphene plasmons, Nature Mater. 16(2), 204-207 (2017).

[169] L. Vicarelli, M. S. Vitiello, D. Coquillat, A. Lombardo, A. C. Ferrari, W. Knap, M. Polini, V. Pellegrini, A. Tredicucci, Graphene field-effect transistors as roomtemperature terahertz detectors, Nature Mater. 11, 865-871 (2012).

[170] R. M. Feenstra, D. Jena, G. Gu, Single-particle tunneling in doped graphene insulator-graphene junctions, $J$. Appl. Phys. 11, 043711 (2012).

[171] V. Ryzhii, A. Satou, T. Otsuji, M. Ryzhii, V. Mitin, M. S. Shur, Dynamic effects in double graphene-layer structures with inter-layer resonant-tunnelling negative conductivity, J. Phys. D: Appl. Phys. 46, 315107 (2013).

[172] A. Mishchenko, J. S. Tu, Y. Cao, R. V. Gorbachev, J. R. Wallbank, M. T. Greenaway, V. E. Morozov, S. V. Morozov, M. J. Zhu, S. L. Wong, F. Withers, C. R. Woods, Y.-J. Kim, K. Watanabe, T. Taniguchi, E. E. Vdovin, O. Makarovsky, T. M. Fromhold, V. I. Fal'ko, A. K. Geim, L. Eaves, K. S. Novoselov, Twist- 
controlled resonant tunnelling in graphene/boron nitride/graphene heterostructures, Nature Nanotechnol. 9, 808-813 (2014).

[173] B. Fallahazad, K. Lee, S. Kang, J. Xue, S. Larentis, C. Corbet, K. Kim, H. C. P. Movva, T. Taniguchi, K. Watanabe, L. F. Register, S. K. Banerjee, E. Tutuc, Gate-tunable resonant tunneling in double bilayer graphene heterostructures, Nano Lett. 15, 428-433 (2014).

[174] A. Tomadin, A. Tredicucci, V. Pellegrini, M. S. Vitiello, M. Polini, Photocurrent-based detection of terahertz radiation in graphene, Appl. Phys. Lett. 103, 211120 (2013).

[175] V. Ryzhii, T. Otsuji, M. Ryzhii, V. Ya Aleshkin, A. A. Dubinov, D. Svintsov, V. Mitin, M. S. Shur, Graphene vertical cascade interband terahertz and infrared photodetectors, 2D Mater. 2(2), 025002 (2015).

[176] B. Sensale-Rodriguez, Graphene-insulator-graphene active plasmonic terahertz devices, Appl. Phys. Lett. 103, 123109 (2013).

[177] A. Zak, M. A. Andersson, M. Bauer, J. Matukas, A. Lisauskas, H. G. Roskos, J. Stake, Antenna-Integrated $0.6 \mathrm{THz}$ FET direct detectors based on CVD graphene, Nano Lett. 14, 5834-5838 (2014).

[178] G. C. Shan, C. H. Shek, and M. J. Hu, Developments of Cavity-Controlled Devices with Graphene and Graphene Nanoribbon for Optoelectronics Applications, Graphene Science Handbook, Chapter 24, pp. 395-410, CRC press, 2016.

[179] W. Knap, S. Rumyantsev, M. S. Vitiello, D. Coquillat, S. Blin, N. Dyakonova, M. Shur, F. Teppe, A. Tredicucci, T. Nagatsuma, Nanometer size field effect transistors for terahertz detectors, Nanotechnology 24, 214002 (2013).

[180] J. C. W. Song, M. S. Rudner, C. M. Marcus, L. S. Levitov, Hot carrier transport and photocurrent response in graphene, Nano Lett. 11, 4688-4692 (2011).

[181] M. Freitag, T. Low, F. Xia, P. Avouris, Photoconductivity of biased graphene, Nat. Photonics 7, 53-59 (2013).

[182] J. Yan, M.-H. Kim, J. A. Elle, A. B. Sushkov, G. S. Jenkins, H. M. Milchberg, M. S. Fuhrer, H. D. Drew, Dual-gated bilayer graphene hot-electron bolometer, Nature Nanotechnol. 7, 472-478 (2012).

[183] X. Cai, A. B. Sushkov, R. J. Suess, M. M. Jadidi, G. S. Jenkins, L. O. Nyakiti, R. L. Myers-Ward, S. Li, J. Yan, D. K. Gaskill, T. E. Murphy, H. D. Drew, M. S. Fuhrer, Sensitive room-temperature terahertz detection via the photothermoelectric effect in graphene, Nature Nanotechnol. 9, 814-819 (2014).

[184] A. V. Muraviev, S. L. Rumyantsev, G. Liu, A. A. Balandin, W. Knap, M. S. Shur, Plasmonic and bolometric terahertz detection by graphene field-effect transistor, Appl. Phys. Lett. 103, 0181114 (2013).

[185] F. Schwierz, Graphene transistors. Nature Nanotechnology 5 (7), 487 (2010).

[186] F. Schwierz, Graphene Transistors: Status, Prospects, and Problems. Proceedings of the Ieee 101 (7), 1567 (2013).

[187] W. Y. Fu, L. Jiang, E. P. van Geest, L. M. C. Lima and G. F. Schneider, Sensing at the Surface of Graphene Field-Effect Transistors. Adv. Mater. 29 (6), (2017).

[188] J. S. Friedman, A. Girdhar, R. M. Gelfand, G. Memik, H. Mohseni, A. Taflove, B. W. Wessels, J. P. Leburton and A. V. Sahakian, Cascaded spintronic logic with lowdimensional carbon. Nat. Commun. 8, 15635 (2017).

[189] E. C. Ahn, H. S. P. Wong and E. Pop, Carbon nanomaterials for non-volatile memories. Nature Reviews Materials 3 (3), (2018).

[190] F. Hui, E. Grustan-Gutierrez, S. B. Long, Q. Liu, A. K. Ott, A. C. Ferrari and M. Lanza, Graphene and Related Materials for Resistive Random Access Memories. Advanced Electronic Materials 3 (8), (2017).

[191] J. Y. Son, Y. H. Shin, H. Kim and H. M. Jang, NiO Resistive Random Access Memory Nanocapacitor Array on Graphene. Acs Nano 4 (5), 2655 (2010).

[192] H. J. Hwang, J. H. Yang, Y. G. Lee, C. Cho, C. G. Kang, S. C. Kang, W. Park and B. H. Lee, Ferroelectric polymer-gated graphene memory with high speed conductivity modulation. Nanotechnology 24 (17), 175202 (2013).

[193] H. Tian, H. Y. Chen, B. Gao, S. Yu, J. Liang, Y. Yang, D. Xie, J. Kang, T. L. Ren, Y. Zhang and H. S. Wong, Monitoring oxygen movement by Raman spectroscopy of resistive random access memory with a grapheneinserted electrode. Nano Lett. 13 (2), 651 (2013).

[194] Y. Ji, S. Lee, B. Cho, S. Song and T. Lee, Flexible Organic Memory Devices with Multilayer Graphene Electrodes. Acs Nano 5 (7), 5995 (2011).

[195] X. Wang, W. Xie and J. B. Xu, Graphene based nonvolatile memory devices. Adv. Mater. 26 (31), 5496 (2014).

[196] A. Nag, A. Mitra and S. C. Mukhopadhyay, Graphene and its sensor-based applications: A review. Sensors and Actuators a-Physical 270, 177 (2018).

[197] C. I. L. Justino, A. R. Gomes, A. C. Freitas, A. C. Duarte and T. A. P. Rocha-Santos, Graphene based sensors and biosensors. TrAC Trends in Analytical Chemistry 91, 53 (2017).

[198] T. Wang, D. Huang, Z. Yang, S. S. Xu, G. L. He, X. L. Li, N. T. Hu, G. L. Yin, D. N. He and L. Y. Zhang, A Review on Graphene-Based Gas/Vapor Sensors with Unique Properties and Potential Applications. NanoMicro Letters 8 (2), 95 (2016).

[199] E. W. Hill, A. Vijayaragahvan and K. Novoselov, Graphene Sensors. IEEE Sensors Journal 11 (12), 3161 (2011).

[200] E. Singh, M. Meyyappan and H. S. Nalwa, Flexible Graphene-Based Wearable Gas and Chemical Sensors. ACS Appl. Mater. Interfaces 9 (40), 34544 (2017).

[201] A. Gutes, B. Hsia, A. Sussman, W. Mickelson, A. Zettl, C. Carraro and R. Maboudian, Graphene decoration with metal nanoparticles: towards easy integration for sensing applications. Nanoscale 4 (2), 438 (2012).

[202] X. H. Li, W. C. H. Choy, X. G. Ren, D. Zhang and H. F. Lu, Highly Intensified Surface Enhanced Raman Scattering by Using Monolayer Graphene as the Nanospacer of Metal Film-Metal Nanoparticle Coupling System. Advanced Functional Materials 24 (21), 3114 (2014).

[203] X. Li, X. Ren, Y. Zhang, W. C. H. Choy and B. Wei, An all-copper plasmonic sandwich system obtained through directly depositing copper NPs on a CVD grown graphene/copper film and its application in SERS. Nanoscale 7 (26), 11291 (2015).

[204] Y. Zhao and Y. W. Zhu, Graphene-based hybrid films for plasmonic sensing. Nanoscale 7 (35), 14561 (2015). 